\title{
Multiple linear regression modelling to predict the stability of polymer-drug solid dispersions: Comparison of the effects of polymers and manufacturing methods on solid dispersion stability
}

Gudrun A Fridgeirsdottir ${ }^{1}$, Robert J Harris ${ }^{2}$, Ian L Dryden ${ }^{3}$, Peter M Fischer $^{1}$, Clive J Roberts ${ }^{1 *}$

${ }^{1}$ School of Pharmacy, University of Nottingham, University Park, Nottingham, United Kingdom

${ }^{2}$ Juniper Pharma Services Ltd, Nottingham Business Park, Nottingham, United Kingdom

${ }^{3}$ School of Mathematical Sciences, University of Nottingham, University Park, Nottingham, United Kingdom

* School of Pharmacy, University Park, Nottingham, NG7 2RD, UK Tel. 01159515101

Fax. 01158467969 clive.roberts@nottingham.ac.uk 


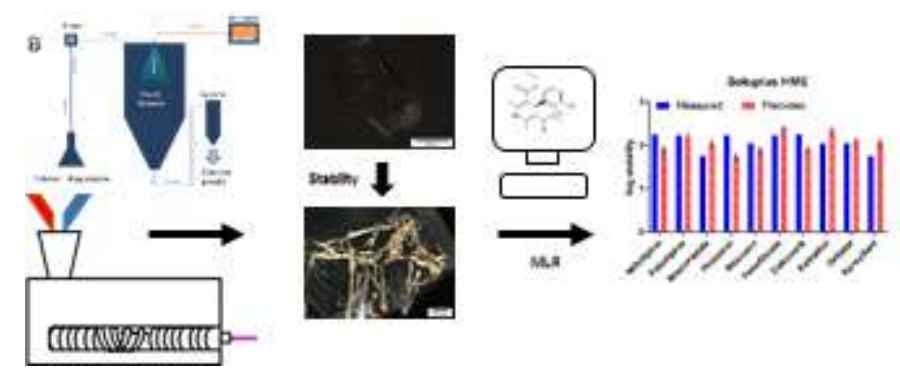

\begin{abstract}
Solid dispersions can be a successful way to enhance the bioavailability of poorly soluble drugs. Here 60 solid dispersion formulations were produced using ten chemically diverse, neutral, poorly soluble drugs, three commonly used polymers, and two manufacturing techniques, spray drying and melt extrusion. Each formulation underwent a six-month stability study at accelerated conditions, $40{ }^{\circ} \mathrm{C}$ and $75 \%$ relative humidity (RH). Significant differences in times to crystallisation (onset of crystallisation) were observed between both the different polymers and the two processing methods. Stability from zero days to over one year was observed. The extensive experimental dataset obtained from this stability study was used to build multiple linear regression models to correlate physicochemical properties of the active pharmaceutical ingredients (API) with the stability data. The purpose of these models is to indicate which combination of processing method and polymer carrier is most likely to give a stable solid dispersion. Six quantitative mathematical multiple linear regression-based models were produced based on selection of the most influential independent physical and chemical parameters from a set of 33 possible factors, one model for each combination of polymer and processing method, with good predictability of stability. Three general rules are proposed from these models for the formulation development of suitably stable solid dispersions. Namely, increased stability is correlated with increased glass transition temperature $\left(T_{\mathrm{g}}\right)$ of solid dispersions, as well as decreased number of H-bond donors and increased molecular flexibility (such as rotatable bonds and ring count) of the drug molecule.
\end{abstract}

Keywords: solid dispersion, stability, multiple linear regression model, hot melt extrusion, spray-drying, amorphous 


\section{INTRODUCTION}

Poor water solubility (and hence bioavailability) of drugs in development is an ongoing and challenging problem, as up to $75 \%$ of new chemical entities (NCE) in small-molecule drug pipelines are poorly water soluble ${ }^{1,2}$. Poor solubility increases the risk of delays and failure in the development of a $\mathrm{NCE}^{3}$. There are several possible routes that can be taken in order to enhance the solubility and bioavailability of drugs through formulation. These include particle size reduction, polymorph optimisation, lipid formulations, and solid dispersions, as reviewed by Williams et al. ${ }^{4}$.

Solid dispersions can significantly increase the effective solubility and thus bioavailability of poorly soluble drugs. This can be accomplished by two strategies; particle size reduction so that the drug is dispersed as tiny particles, or molecular dispersion in a soluble polymeric carrier. The drug is also, if in particulate form, usually dispersed in an amorphous form, which gives increased solubility compared to the crystalline form ${ }^{5-9}$. As the amorphous form is generally unstable, it is likely to convert to the more stable crystalline form, which in turn reduces solubility. Other processes and factors can also contribute to instability within a solid dispersion, inclusive of phase segregation leading to regions with different compositions, chemical degradation of the API leading to products that may alter crystallisation tendencies and water uptake by the matrix polymer that can affect drug and polymer mobility and hence stability ${ }^{10}$. Physical stability of solid dispersions is therefore the most significant obstacle that must be overcome in their development. Choosing an appropriate polymer and processing method is pivotal in producing a successful product as it will greatly affect the stability of the solid dispersions. The most commonly used manufacturing methods are spray-drying and hot melt extrusion (HME) as these are relatively easy to scale up and produce well mixed dispersions ${ }^{11,12}$. The chosen polymer carrier stabilises both the drug in the solid form and in the solid-solution, retarding crystallisation ${ }^{7,13}$. Solid dispersions have been extensively studied in this context (reviewed by Leuner and Dressman ${ }^{14}$ ). Solid dispersions have been used successfully to enhance the bioavailability of drugs in several marketed products as summarised in Table 1.

TABLE 1. Summary of marketed solid dispersions in oral dosage forms, including the brand name, the APIs they contain and the polymer carriers used ${ }^{7,15,16}$. The following abbreviations are used: PEG - polyethylene glycol, PVP - polyvinylpyrrolidone, HPMC - hydroxypropyl methylcellulose, HPMCAS - hydroxypropyl methylcellulose acetate succinate, HPC - hydroxypropyl cellulose.

\begin{tabular}{lll}
\hline Brand name & API & Polymer carrier \\
\hline Adalat SL & Nifedipine & PEG \\
Afeditab CR & Nifedipine & Poloxamer-PVP \\
Certican & Everolimus & HPMC \\
Cesamet & Nabilone & PVP \\
Crestor & Rovustatin & HPMC \\
Envarsus & Tacrolimus & HPMC \\
Fenoglide & Fenofibrate & PEG \\
Gris-PEG & Griseofulvin & PEG \\
Incivo & Telaprevir & HPMCAS \\
Intelence & Etravirine & HPMC \\
Isoptin SR-E & Verapamil & HPC/HPMC \\
Kaletra & Lopinavir/Ritonavir & PVP-VA \\
Kalydeco & Ivacaftor & HPMCAS \\
\hline
\end{tabular}




\begin{tabular}{lll}
\hline Lopinavir/Ritonavir Mylan & Lopinavir /Ritonavir & HPMCAS \\
Modigraf & Tacrolimus & HPMC \\
\hline Nivadil & Nivaldipine & HPMC \\
\hline Noxafil & Posaconazole & HPMCAS \\
\hline Orkambi & Lumacaftor / Ivacaftor & HPMCAS \\
\hline Prograf & Tacrolimus & HPMC \\
Rezulin & Troglitazone & HPMC \\
Sporanox & Itraconazole & HPMC \\
Viekirax & Ombitasvir/Paritaprevir/Ritonavir & PVP-VA \\
Votubia & Everolimus & HPMC \\
Zelboraf & Vemurafenib & HPMCAS \\
\hline
\end{tabular}

Choosing the most appropriate formulation route for a new drug is to a large extent still based on formulator know-how and experimental screening assays. Properties of the Active Pharmaceutical Ingredient (API) are often not considered, but rather in-house knowledge and availability of equipment steer the process ${ }^{17,18}$. We propose that a knowledge-based computational tool that could predict the best formulation strategy would greatly improve this process. Indeed there are some support tools available that can be used to facilitate this process such as guidance maps, high through-put screening, and statistical models, as we recently reviewed ${ }^{19}$. Using a support tool in the formulation development can shorten the development process, limiting the number of experiments that need to be carried out by indicating which formulation route is appropriate for a specific API and there remains a significant need in this area.

As the choice of polymer carrier and processing method are vital to the consequent stability of a solid dispersion, the use of a knowledge-based support tool in the formulation development is very beneficial. Currently, although some effort has been put into building such tools, their availability remains quite limited ${ }^{18,20}$. Several models have been built to predict the glassforming ability of APIs and some predict the stability of the amorphous form of APIs. These are intended to give an indication of whether a dosage form containing the API in amorphous form is a viable formulation choice ${ }^{21-26}$. Recently molecular dynamics (MD) simulations have been used to predict $T_{\mathrm{g}}$, investigate interactions between drug and polymer in solid dispersions, and the effect of water in the system ${ }^{27}$. Historically the Flory-Huggins equation or Hansen solubility parameters have also been used theoretically to analyse interactions and miscibility and provide an indication of likely stability. The advancement of a computational tool that can compare several solid dispersion formulations and predict their stability has not yet been achieved.

Many researchers have focused on the stability of solid dispersions in their studies, in particular which factors inhibit crystallisation ${ }^{28-31}$. With better knowledge of which factors are important for stabilisation, such as drug-polymer interaction, polymer $T_{g}$ and hygroscopicity, formulation development of solid dispersions could be improved. This in turn could ease the path of such new formulations to the market. However, although many drugs and polymers have been researched, the number of APIs in such studies is often very limited. This, and the fact that there is little agreement in results between different studies, makes it difficult to draw any general conclusions, as has been noted before ${ }^{32}$. Therefore, the aim of the present study is to produce and test the stability of a large number (60) of solid dispersions using chemically 
diverse APIs (10), commonly used polymers (3) and well-known processing methods, spray drying and melt-extrusion. This approach should yield more general conclusions about important factors for solid dispersions physical stability. The dataset was then used to build a statistical predictive model that should be able to highlight which physicochemical properties of the APIs are correlated with formulation stability. The models could then be used to facilitate formulation development of solid dispersions by indicating which combination of polymer and processing method is most suitable for a new drug.

\section{MATERIALS AND METHODS}

Ten poorly soluble, neutral ( $\mathrm{pKa}_{\mathrm{Acid}}>8.5$ and $\mathrm{pKa} \mathrm{Basic}<4.5$ ), chemically diverse APIs (purity 99\% unless otherwise indicated): nifedipine (1), felodipine (2), itraconazole (3), probucol (4), ritonavir (5), fenofibrate (6), celecoxib (7), estradiol (8), orlistat (9), and aprepitant (10) (purity 98\%), see Figure 2, were purchased from Seqouia Research Products Ltd. Three polymers were generous gifts from their commercial suppliers: Soluplus (PCL-PVAc-PEG, SOL) and Kollidon VA64 (PVP-VA) from BASF, AquaSolve (HPMCAS-HG)) from Ashland (Figure 1). The molecular weight distribution of the three polymers was as follows, $45.000-70.000 \mathrm{Da}$ for PVP-VA, 55.000-90.000 Da for HPMCAS and 90.000-140.000 Da for SOL. Binary solid dispersions were made for each combination of polymer and drug. Each combination was produced twice, once using hot melt extrusion (HME) and once by spray-drying. Each formulation was then subjected to a stability test and characterised at each time point. The following abbreviations of formulations are used below, numbers one to ten for the APIs as listed above (Figure 2). For the polymers S, P and H for Soluplus, PVP-VA, and HPMCAS, respectively. Hot melt extrusion (h) and spray-drying (s). Thus e.g. spray-dried ritonavir PVP-VA is abbreviated 5Ps.
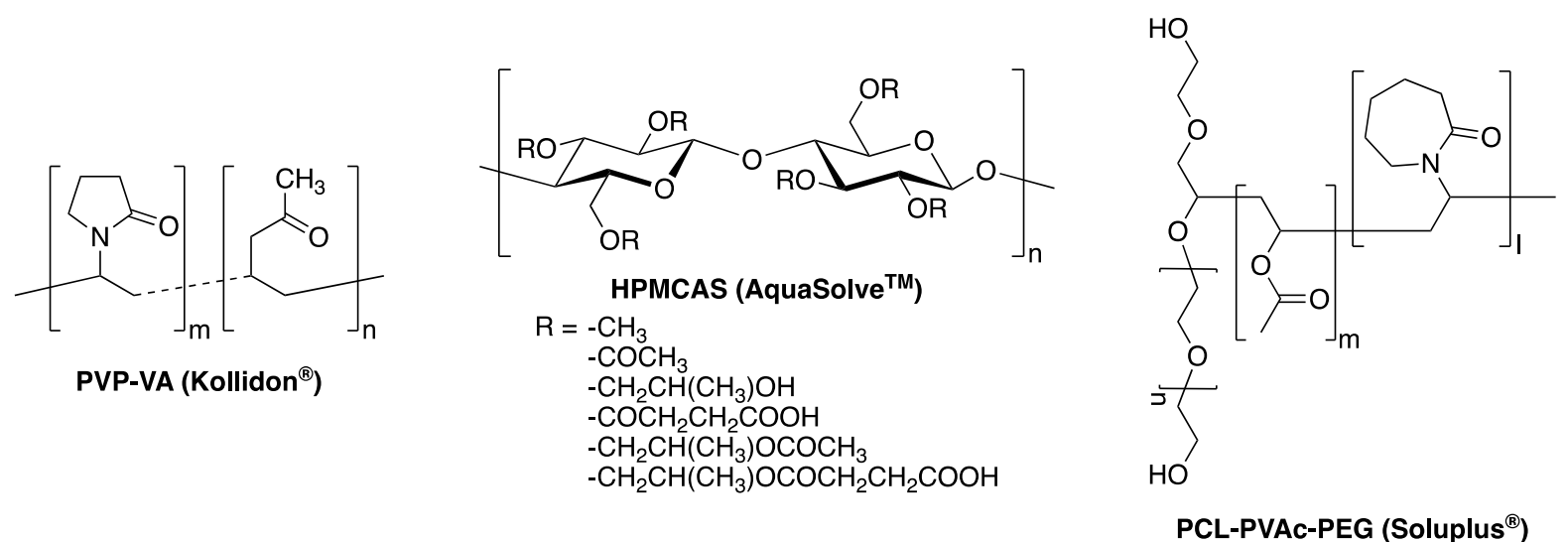

Figure 1 The chemical structure of PVP-VA (P), HPMCAS (H), and SOL (S). 

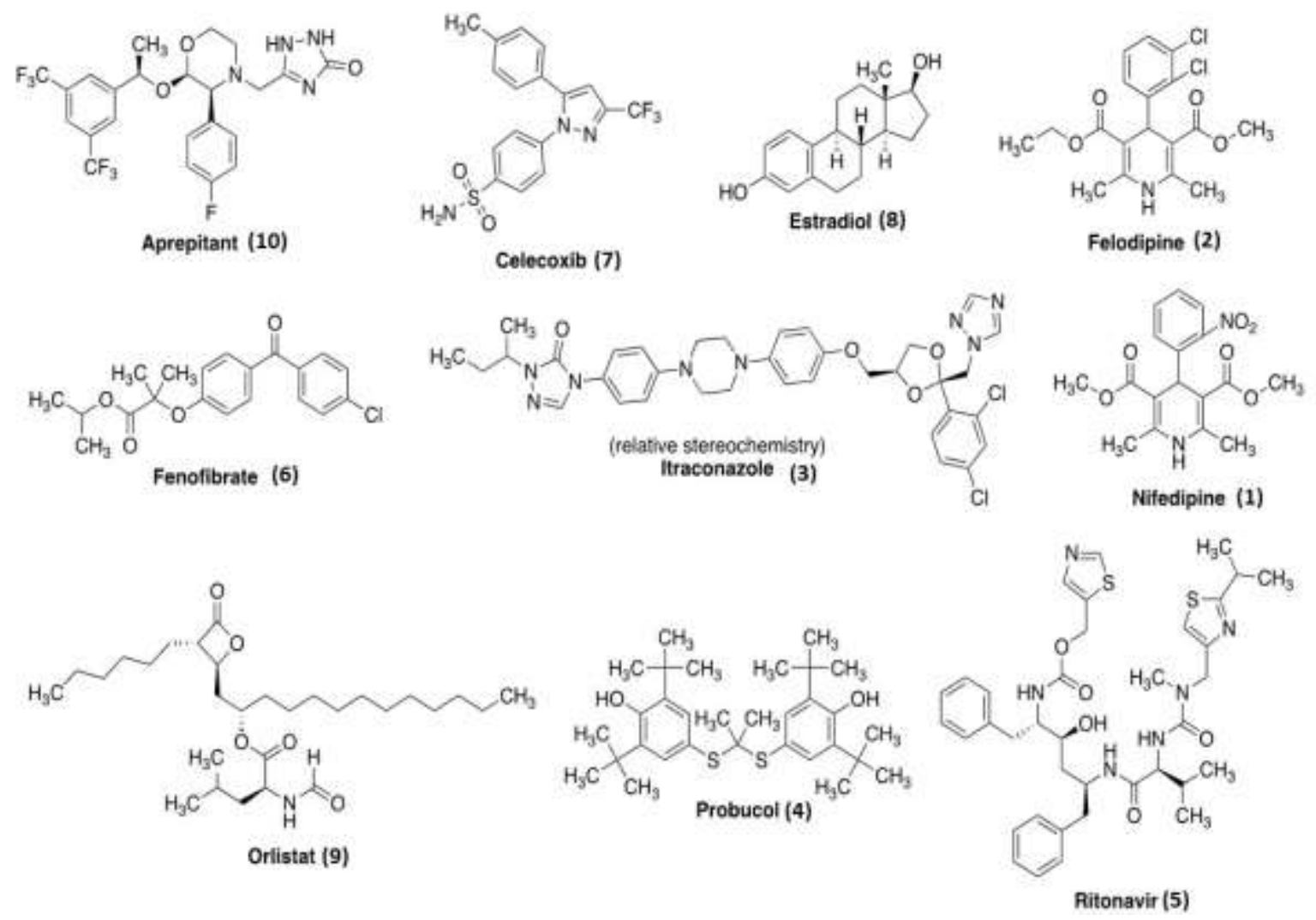

Figure 2 Chemical structure of the ten APIs used in this study.

\section{HOT MELT EXTRUSION}

A HAAKE minilab II micro compounder (Thermo Fisher Scientific) was used for the production of the HME solid dispersions. All formulations were made with $10 \% \mathrm{w} / \mathrm{w}$ drug loading in polymer. In order to keep the number of variables as low as possible all solid dispersions were produced using the same method and no optimisation was performed for any formulation. API and polymer were weighed and mixed in a scintillation vial. Once the minilab had reached the set temperature of $160^{\circ} \mathrm{C}$, the mixture was fed into the hopper. The twin screws were co-rotated at 50 r.p.m. and the mixture was circulated for $15 \mathrm{~min}$ before being extruded. The same method was used for all formulations except those containing orlistat, which was not circulated but extruded immediately because of its low melting point $\left(44{ }^{\circ} \mathrm{C}\right)$. The processing temperature of $160{ }^{\circ} \mathrm{C}$ was selected as an optimal value suitable for the processing of all the selected materials and one that did not cause degradation of the materials during the exposure to this temperature. This processing temperature of is above the $\mathrm{Tg}$ of all three polymers. Therefore, for the three APIs with a melting points above $160^{\circ} \mathrm{C}$ as long as they are miscible in the polymer they should dissolve in the polymer and be fully amorphisised and mixed in the solid dispersion. The success of this approach, or not, would be apparent from studies of the melt extruded mixtures. These did not result in markedly less stabile formulations than those APIs with lower mp. Once extruded, the formulation was placed into amber HPLC vials and stored at $5{ }^{\circ} \mathrm{C} / 0 \% \mathrm{RH}$ until the start of the stability study. 


\section{SPRAY-DRYING}

As with the HME formulations, to keep the number of variables at a minimum, the same method was used to produce all formulations. All were produced using the same solvent, except itraconazole that was not soluble in methanol and was therefore dissolved in dichloromethane. Spray-drying was performed using a Büchi B290 instrument. Each drug-polymer mixture was dissolved in methanol at $10 \% \mathrm{w} / \mathrm{v}$ and spray-dried within half an hour. Once the mixture was fully dissolved in the solvent the solution was sprayed through the nozzle using an inlet temperature of around $80{ }^{\circ} \mathrm{C}$ to maintain an outlet temperature of $50{ }^{\circ} \mathrm{C}$. The produced formulation was then collected and weighed before being placed in amber HPLC vials and stored at $5{ }^{\circ} \mathrm{C} / 0 \% \mathrm{RH}$ until the stability study was started.

\section{STABILITY}

A six-month stability study was conducted on all produced solid dispersions at accelerated conditions, $40{ }^{\circ} \mathrm{C} / 75 \% \mathrm{RH}$, in accordance with $\mathrm{ICH}$ guidelines ${ }^{33}$. The samples were kept glass vials, open containers and conditions were monitored using a Testo $174 \mathrm{H}$ temperature and humidity logger. The amorphicity of the formulations was confirmed at the start of the stability study. Subsequently the solid dispersions were analysed at 12 h, $7 \mathrm{~d}, 1$ month, 2 months, 4 months and 6 months. For those solid dispersions that were still amorphous after 6 months, a 1-year time point was added. At each of these time points the samples were examined for signs of crystallinity using polarised light microscopy (PLM) as a sensitive method to detect onset of crystallisation. Any formulations with signs of crystallinity by PLM were then further characterised using differential scanning calorimetry (DSC) and X-ray powder diffraction (XRPD). Before analysing the samples at each time point the formulation was ground in a mortar, if necessary, producing small glass-like pieces appropriate for characterisation with PLM, DSC, and XRPD.

\section{POLARISED LIGHT MICROSCOPY}

An Olympus BX51 optical microscope fitted with polarising filters and equipped with an Olympus DP71 camera was used visually to check for presence of any crystalline material. A small amount of powder (spray-dried) or ground glass (HME) was placed on a microscope slide with a glass cover slip. The samples were examined using both cross-polarised and nonpolarised light. Images of identical frames were recorded for each sample under both polarised and non-polarised light. Cell ${ }^{\wedge} \mathrm{F}$ imaging system from Olympus was used to acquire images of the samples.

\section{DIFFERENTIAL SCANNING CALORIMETRY}

A Q2000 (TA instruments) was used for the DSC measurements of all samples, using Tzero pans (TA instruments) and pierced lids. The instrument was calibrated against indium. Approximately $5 \mathrm{mg}$ of sample was weighed into each pan. Each sample was run in duplicate. All solid dispersions, except orlistat, were run as a cycle where the first ramp was used to remove water and solvent from the formulation. The second time the sample was ramped up to $20{ }^{\circ} \mathrm{C}$ above the melting point of the drug. Both up and down ramps were performed at 10 ${ }^{\circ} \mathrm{C} / \mathrm{min}$. As orlistat has a melting point of $44{ }^{\circ} \mathrm{C}$ it was not feasible to go above $100^{\circ} \mathrm{C}$ in order 
to remove water before running the sample as this might have affected the solid dispersion. It was therefore run as a single ramp. The results were analysed using the TA Universal Analysis programme to determine $T_{\mathrm{g}}$ and melting points ( $\mathrm{mp}$ ).

\section{X-RAY POWDER DIFFRACTION}

A Bruker D8 Advance instrument using a $\mathrm{Cu} \mathrm{Kal}$ radiation source and equipped with an incident beam monochromator was used for the XRPD measurements. The instrument was calibrated against corundum. All samples were analysed with spinning at a current of $40 \mathrm{~mA}$ and a voltage of $40 \mathrm{kV}$ using standard scanning parameters, i.e. from 2 to $40^{\circ}(2 \theta)$ using a step size of $0.02^{\circ}$ and a time per step of $1 \mathrm{~s}$. A zero-background holder was used for the measurements of samples of approximately 3-5 mg. The program DIFFRAC.SUIT EVA from Bruker was used to analyse and plot the data, no adjustments were done to the baseline during plotting.

\section{PHYSICOCHEMICAL PROPERTIES OF APIS}

The program Instant JChem from ChemAxon was used to calculate and predict physicochemical properties of the APIs. These were based on the molecular structure of each API. Furthermore information on the melting point ( $\mathrm{mp}$ ) of each drug was obtained from Chemspider, these did not differ significantly from measured $\mathrm{mp}^{34}$. The following parameters were included in the modelling process: molecular weight ( $\mathrm{MW})$, lipophilicity (ClogP) determined using the ChemAxon method, strongest basic $\mathrm{p} K_{\mathrm{a}}$ (b.pKa), topological polar surface area (tPSA), number of atoms (nAtom), number of bonds (nBond), number of chiral atoms (nChir), number of $\mathrm{H}$-bond acceptors (HBA), number of $\mathrm{H}$-bond donors (HBD), number of rings (nRing), number of nonterminal rotatable bonds (nRot), number of aliphatic atoms (nAliph), number of aromatic atoms (nAr), water accessible surface area (ASA), average polarizability (avPol), hydrophilicity-lipophilicity balance (HLB), intrinsic water solubility in $\log _{10}$ of molar solubility $\left(\log \mathrm{S}_{0}\right)$, molecular polarizability (molPol), van der Waals surface area (vdW-SA), melting point (mp), number of aliphatic rings (nAliphRing), number of aromatic rings (nAr), number of carbon atoms $(\mathrm{nC})$, number of fluorine $(\mathrm{nF})$ atoms, number of nitrogen atoms $(\mathrm{nN})$, number of oxygen atoms $(\mathrm{nO})$, number of sulphur atoms $(\mathrm{nS})$, number of chlorine atoms $(\mathrm{nCl})$, number of heteroatoms (atoms other than carbon and hydrogen; $\mathrm{nHet}$ ), number of $\mathrm{sp}^{3}$ carbon atoms, number of $\mathrm{sp}^{2}$ carbon atoms, measured $T_{\mathrm{g}}\left(T_{g, \text { meas }}\right)$ and predicted $T_{\mathrm{g}}\left(T_{g, \text { pred }}\right)$ in ${ }^{\circ} \mathrm{C}$, where the Fox equation was used to calculate the expected $T_{\mathrm{g}}$ of the solid dispersion. The predicted value was also used as a $T_{g \text {,meas }}$, in those instances where a $T_{\mathrm{g}}$ could not be measured. The physicochemical properties used and their values are listed in Table 2. 
TABLE 2 Calculated and predicted parameters used in the building of the MLR models.

\begin{tabular}{|c|c|c|c|c|c|c|c|c|c|c|}
\hline $\begin{array}{r}\text { Physicochemical } \\
\text { property }\end{array}$ & 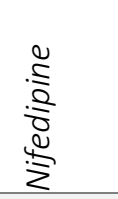 & $\begin{array}{l}\frac{0}{a} \\
\frac{0}{0} \\
\frac{0}{2} \\
L\end{array}$ & 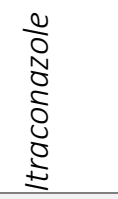 & $\begin{array}{l}\bar{g} \\
\bar{z} \\
\bar{d} \\
\bar{d}\end{array}$ & $\begin{array}{l}\frac{1}{3} \\
\frac{0}{0} \\
\frac{0}{0} \\
\frac{0}{\alpha}\end{array}$ & 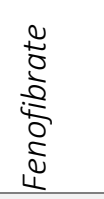 & 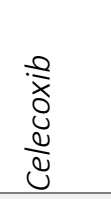 & $\begin{array}{l}\bar{o} \\
\overline{\bar{o}} \\
\overline{0} \\
\overline{\mathrm{D}}\end{array}$ & 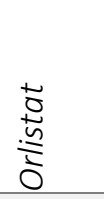 & $\begin{array}{l}\frac{a}{5} \\
\frac{ \pm}{0} \\
\frac{a}{2} \\
\frac{a}{2}\end{array}$ \\
\hline nAliph & 19 & 19 & 21 & 23 & 28 & 13 & 9 & 14 & 35 & 20 \\
\hline nAliphRing & 1 & 1 & 2 & 0 & 0 & 0 & 0 & 3 & 1 & 1 \\
\hline$n A r$ & 6 & 6 & 28 & 12 & 22 & 12 & 17 & 6 & 0 & 17 \\
\hline nArRing & 1 & 1 & 5 & 2 & 4 & 2 & 3 & 1 & 0 & 3 \\
\hline$A S A\left(\AA^{2}\right)$ & 533 & 583 & 976 & 874 & 981 & 642 & 602 & 392 & 985 & 679 \\
\hline nAtom & 43 & 44 & 87 & 83 & 98 & 46 & 40 & 44 & 88 & 58 \\
\hline $\operatorname{avPol}\left(\AA^{3}\right)$ & 33 & 37 & 74 & 62 & 76 & 38 & 35 & 32 & 61 & 44 \\
\hline nBond & 44 & 45 & 93 & 84 & 101 & 47 & 42 & 47 & 88 & 61 \\
\hline nChir & 0 & 1 & 3 & 0 & 4 & 0 & 0 & 5 & 4 & 3 \\
\hline$H B A$ & 5 & 3 & 9 & 2 & 6 & 3 & 3 & 2 & 3 & 6 \\
\hline$H B D$ & 1 & 1 & 0 & 2 & 4 & 0 & 1 & 2 & 1 & 2 \\
\hline$n$ Het & 8 & 7 & 14 & 4 & 13 & 5 & 9 & 2 & 6 & 14 \\
\hline$H L B$ & 16 & 11 & 5 & 2 & 4 & 4 & 4 & 2 & 3 & 16 \\
\hline $\log S_{0}(M)$ & -3 & -4 & -9 & -11 & -8 & -6 & -6 & -4 & -11 & -6 \\
\hline $\log P$ & 1.82 & 3.44 & 7.31 & 10.57 & 5.22 & 5.28 & 4.01 & 3.75 & 8.11 & 4.52 \\
\hline$M W$ & 346.3 & 384.3 & 705.6 & 519.8 & 721.0 & 360.8 & 381.4 & 272.4 & 495.8 & 534.4 \\
\hline $\operatorname{molPol}\left(\AA^{3}\right)$ & 33 & 37 & 71 & 61 & 75 & 38 & 36 & 31 & 56 & 43 \\
\hline$m p\left({ }^{\circ} \mathrm{C}\right)$ & 173 & 145 & 166 & 125 & 121 & 81 & 158 & 152 & 44 & 255 \\
\hline$n C$ & 17 & 18 & 35 & 31 & 37 & 20 & 17 & 18 & 29 & 23 \\
\hline $\mathrm{nCl}$ & 0 & 2 & 2 & 0 & 0 & 1 & 0 & 0 & 0 & 0 \\
\hline$n F$ & 0 & 0 & 0 & 0 & 0 & 0 & 3 & 0 & 0 & 7 \\
\hline$n N$ & 2 & 1 & 8 & 0 & 6 & 0 & 3 & 0 & 1 & 4 \\
\hline no & 6 & 4 & 4 & 2 & 5 & 4 & 2 & 2 & 5 & 3 \\
\hline$n S$ & 0 & 0 & 0 & 2 & 2 & 0 & 1 & 0 & 0 & 0 \\
\hline nRing & 2 & 2 & 7 & 2 & 4 & 2 & 3 & 4 & 1 & 4 \\
\hline$n R o t$ & 6 & 6 & 11 & 8 & 18 & 7 & 4 & 0 & 23 & 8 \\
\hline$s p^{2}$ & 12 & 12 & 22 & 12 & 21 & 14 & 15 & 6 & 3 & 14 \\
\hline$s p^{3}$ & 5 & 6 & 13 & 19 & 16 & 6 & 2 & 12 & 26 & 9 \\
\hline b.p $p K_{a}$ & -6.63 & -6.64 & 3.92 & -5.08 & 2.84 & -4.93 & 0.08 & -0.88 & -1.44 & 3.74 \\
\hline $\operatorname{tPSA}\left(\AA^{2}\right)$ & 107.8 & 64.6 & 100.8 & 40.5 & 145.8 & 52.6 & 78.0 & 40.5 & 81.7 & 75.2 \\
\hline$v d W-S A\left(\AA^{2}\right)$ & 466 & 493 & 965 & 916 & 1,065 & 533 & 484 & 437 & 925 & 656 \\
\hline $\operatorname{Sh} T_{\text {g meas }}\left({ }^{\circ} \mathrm{C}\right)$ & 70.2 & 68.2 & 68.5 & 68.9 & 66.6 & 56.1 & 72.7 & 64.2 & NA & 74.3 \\
\hline Ss $T_{g \text { meas }}\left({ }^{\circ} \mathrm{C}\right)$ & 71.2 & 67.8 & 70.0 & 67.0 & 68.3 & 57.5 & 71.7 & 68.0 & NA & 74.2 \\
\hline$P h T_{\text {g meas }}\left({ }^{\circ} \mathrm{C}\right)$ & 102.3 & 100.9 & 103.6 & 101.4 & 96.3 & 84.8 & 104.8 & 103.1 & NA & 106.0 \\
\hline Ps $T_{\text {g meas }}\left({ }^{\circ} \mathrm{C}\right)$ & 104.8 & 104.5 & 109.9 & 103.4 & 102.5 & 91.8 & 103.7 & 99.5 & NA & 110.6 \\
\hline$H h T_{\text {g meas }}\left({ }^{\circ} \mathrm{C}\right)$ & 105.3 & 103.4 & 109.0 & 101.7 & 97.6 & 92.7 & 102.0 & 104.7 & NA & NA \\
\hline$H s T_{g}$ meas $\left({ }^{\circ} \mathrm{C}\right)$ & 103.7 & 103.7 & 109.3 & 104.3 & 102.6 & 91.1 & 103.7 & 100.0 & 94.8 & 110.0 \\
\hline$S T_{g \text { pred }}\left({ }^{\circ} \mathrm{C}\right)$ & 67.5 & 67.5 & 68.8 & 65.7 & 67.9 & 61.1 & 68.8 & 71.5 & 58.5 & 66.6 \\
\hline$P T_{g \text { pred }}\left({ }^{\circ} \mathrm{C}\right)$ & 95.4 & 95.4 & 96.7 & 93.6 & 95.8 & 89.0 & 96.7 & 99.4 & 86.4 & 94.5 \\
\hline
\end{tabular}




\section{MULTIPLE LINEAR REGRESSION (MLR) MODEL}

The publicly available software $\mathrm{R}^{35}$ and $\mathrm{R}$ studio (version 1.0.143) were used to build the multiple linear regression models. The calculated and predicted parameters (see Table 2) were used without units as input variables in the model equations. The measured stability (time to crystallisation), the output, was used as the $\log _{10}$ value, stability in days +1 in order to avoid zero values. Using the leaps package in $\mathrm{R}$ an exhaustive selection of covariates was made with one, two and three as a maximum number of covariates; using backwards, forwards, stepwise, and sequential replacement. This was done to insure that the simplest, most effective model could be made, so that each parameter improves the model more than can be expected by chance. To avoid linear dependency of covariates, the selected parameters were tested for intercorrelation and further iterations of parameter selection were made if this was found to be higher than 0.9. Once three uncorrelated parameters had been selected, these were used to build a model that linked the physicochemical properties with the logarithm of the measured stability, i.e. time until onset of crystallisation. As this was a relatively limited dataset (in terms of statistical analysis) of ten observation per model, the whole dataset was used to build the models, rather than a training and test set method. A leave-one-out cross validation (LOOCV) was performed on each model to test its predictability and to calculate the mean square error (MSE). This process was repeated for each dataset (combination of polymer and processing method), resulting in six MLR models.

\section{RESULTS}

Due to limit of detection of XRPD and DSC, PLM was used as the main method of detecting onset of crystallisation, while further crystallisation was confirmed using the inherently more quantitative XRPD and DSC methods. In many cases, even when an abundance of crystals was observed by PLM, no crystallinity could be detected with either XRPD or DSC (Figure 3). 

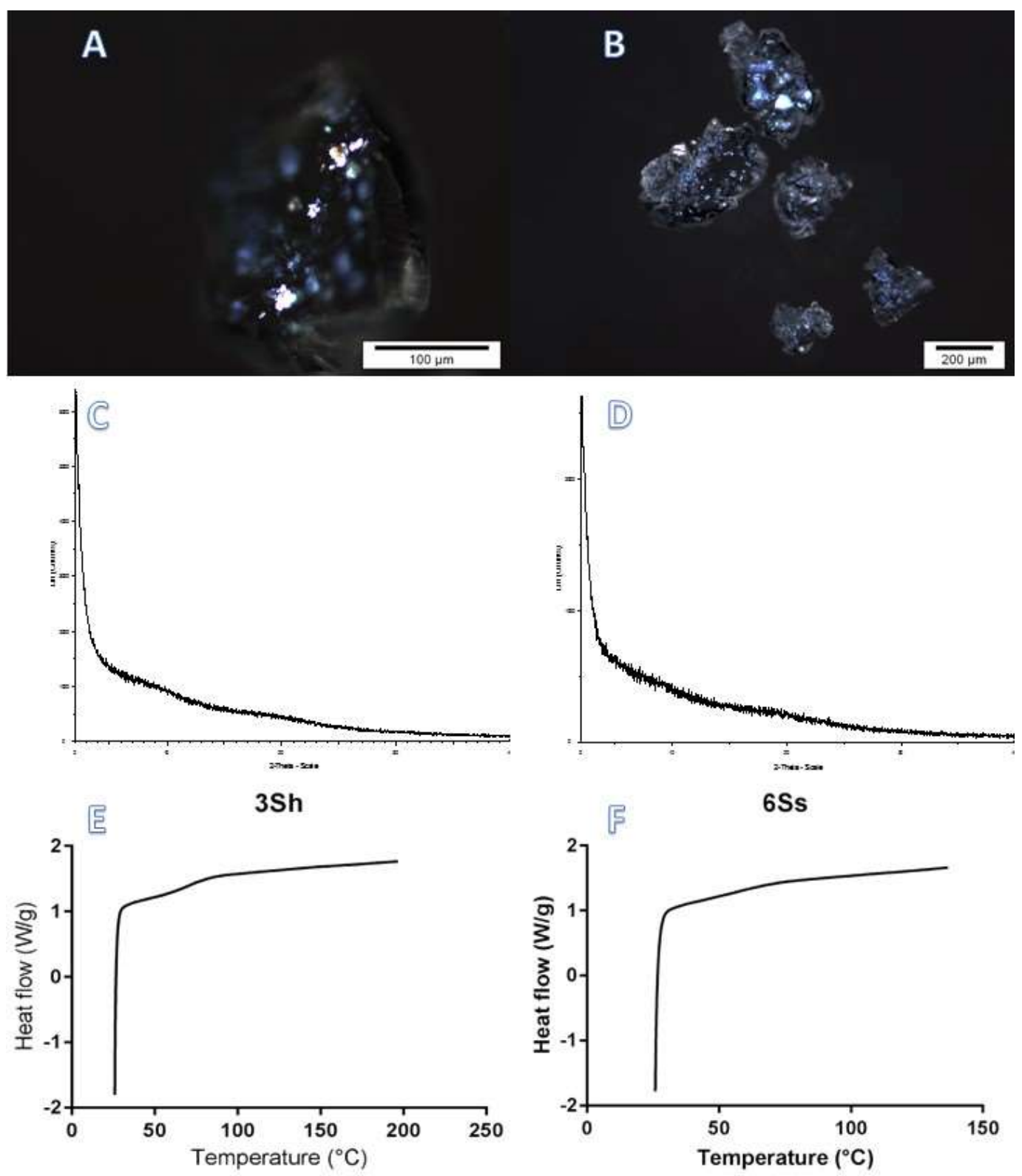

FIGURE 3 A) 3Sh with clearly visible crystals under polarised light, C) and D) no melting peak is visible under DSC nor any peaks in XRPD. B) 6Ss with abundance of crystals visible, again no melting peak by DSC nor peaks indicating crystallinity in $\mathrm{XRPD}, \mathbf{D})$ and $\mathbf{F})$.

\section{STABILITY}

For each API-polymer mixture the HME solid dispersion appeared as a clear glass after extrusion, an indication of amorphicity, except $10 \mathrm{Hh}$, which was cloudy. At the start of the stability assessment all the clear glass HME formulations and all the spray-dried formulations for all APIs were confirmed to be amorphous, while the cloudy $10 \mathrm{Hh}$ formulations were confirmed to contain crystals. Crystals were defined as bright white or coloured spots under polarised light. In each case it was ascertained that spots in the PLM images displayed a distinctive pattern that was not due to any physical defects of the surfaces of the examined 
samples, such as cracks and sharp edges (Figure 4). Even though the onset of crystallisation was the main outcome of this study, once a formulation showed signs of crystallinity, during the stability study, it was followed up at each time point after onset to confirm that further crystallisation had occurred and that the first onset point was not an artefact of measurement.

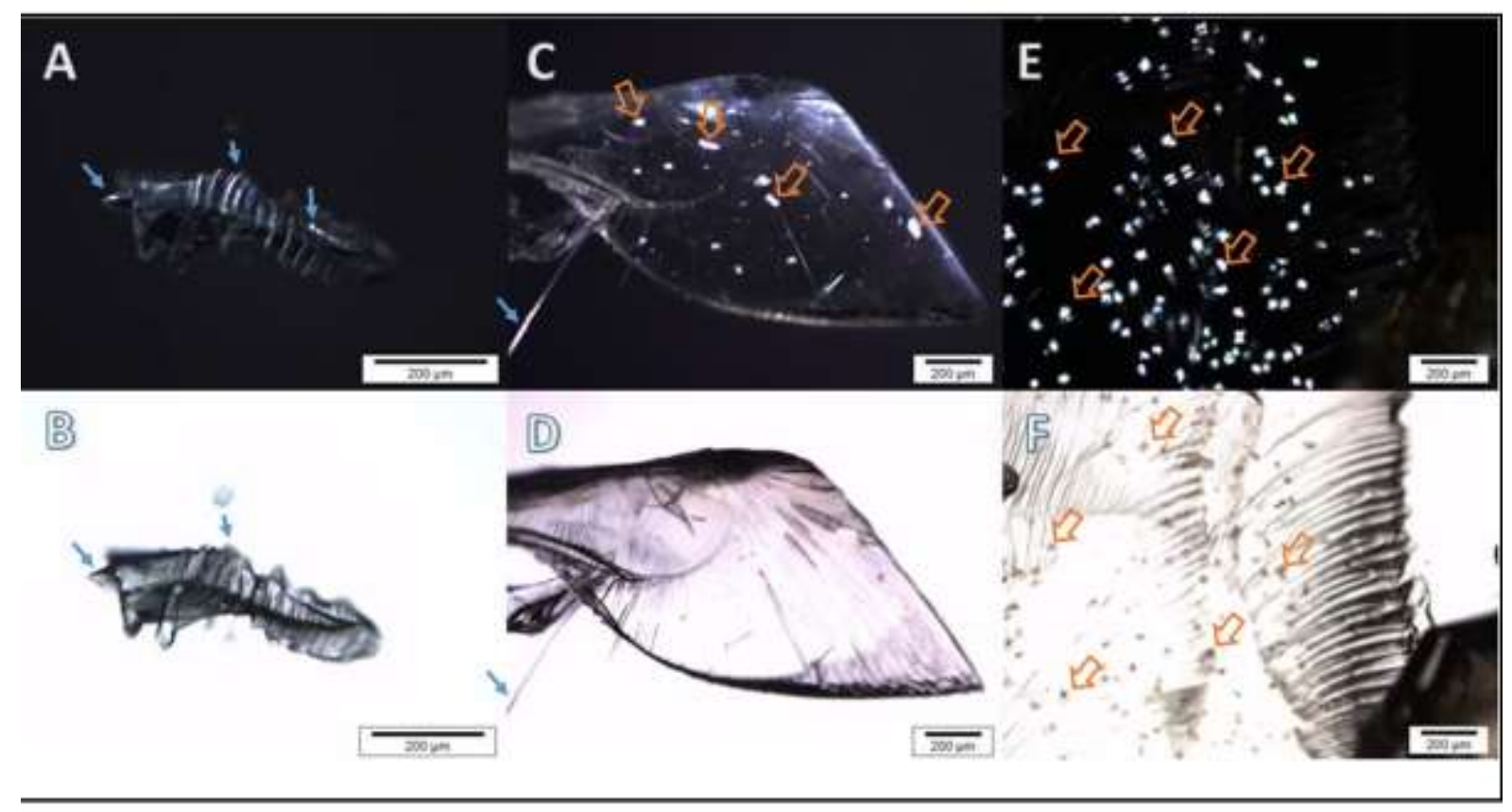

FIGURE 4. Pieces of HME formulations under polarised light (A, C and E) and non-polarised light (B, D and F). (A) 8Ph sharp edges (blue, thin arrows) are visible under polarised light, the visible lines are confirmed as part of the glass rather than crystals (B). Panels $\mathbf{C}$ and $\mathbf{D}$ show visible crystals (orange, thick arrows) under polarised light, 9Hh and 8Sh respectively. No sign of crystals can be seen under normal light in $\mathbf{D}$ but crystals are clearly observed in $\mathbf{F}$.

At the start of the stability study the spray-dried solid dispersions were dispensed into the storage vials as dry dispersible powders but after a period stored at $40{ }^{\circ} \mathrm{C} / 75 \% \mathrm{RH}$ most of the powders had converted to solid glasses, similar in appearance to the HME glass. In most cases signs of crystallisation were not seen until after the transformation of the powder to the glass. How fast this transformation was observed depended on both the polymers and the APIs as some solid dispersions were more resistant. The first transformation was seen after one month at accelerated conditions for 5Ss, 6Ss and 9Ss. Most appeared after two months and a few after four months, 3Ps, 7Ps and 10Ps. The only formulation not to show any signs of transformation during the one year stability study, of those containing PVP-VA and SOL was 4Ss. An example of this transformation can be seen in Figure 5 where the first image shows a well-dispersed powder; panels $\mathrm{C}$ and $\mathrm{D}$ shows a piece where signs of merged particles can be seen but in the last image no signs of previous particles are observed. This transformation to glass was not observed under less accelerated conditions and not for any of the HPMCAS spray-dried formulations. 


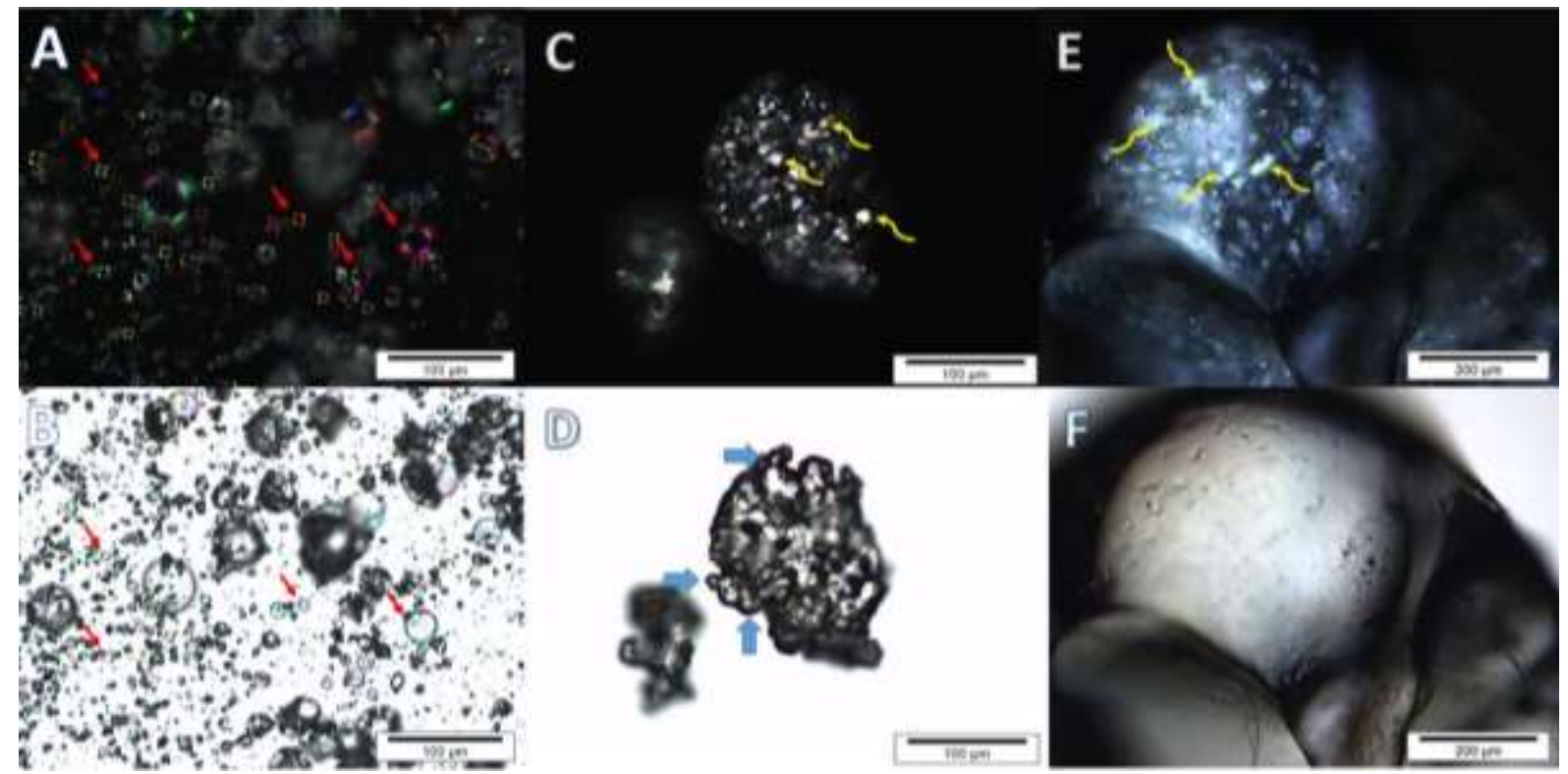

FIGURE 5. Panels A, $\mathbf{C}$, and $\mathbf{E}$ show solid dispersions under cross-polarised light while panels $\mathbf{B}, \mathbf{D}$, and $\mathbf{F}$ show the same samples imaged under normal light. Panels $\mathbf{A}$ and $\mathbf{B}$ show a well-dispersed powder, 6Hs, where typical bubble-like particles (red, thin arrows) can be observed. Panels $\mathbf{C}$ and $\mathbf{D}$ show a piece of 6Ps where the powder has transformed into glass. It is possible to still see signs of the original particles that have merged (blue, thick arrows). Panels $\mathbf{E}$ and $\mathbf{F}$ show a large piece of 6Ss formulation. Here the transformation into glass is more complete than in (D) as no signs of previous particles can be observed. Crystallisation can be observed (yellow, curvy arrows) in $\mathbf{C}$ and $\mathbf{E}$.

The onset of crystallisation was noted for each formulation. For those solid dispersions where no signs of crystals could be found after 6 months at $40{ }^{\circ} \mathrm{C} / 75 \% \mathrm{RH}$ a 1 -year time point was added. In Figure 6 the stability, i.e. the last time point where no signs of crystals were observed, is summarised for each API-polymer combination. For example, if crystals were first observed at 4 weeks, crystals must have started growing between time points 1 week and 4 weeks. Thus the last data point of confirmed amorphicity is 1 week and that is the noted stability.

An overview of the difference in stability is provided in Figure 6. Significant differences in stability between the polymers can be observed for some APIs. As an example, the last amorphous time point for felodipine was observed as 1 weeks in the HPMCAS HME formulation, at 16 weeks when formulated with PVP-VA by HME and at 24 weeks with SOL by HME. A similar example can be found in the spray-dried formulations, probucol was only stable for 7 days in PVP-VA, for a moderate 111 days in HPMCAS but was still amorphous at the one-year point (379 days) in SOL. Some similarities are also observed between the polymers, aprepitant seems to be the least stable of all the drugs by HME regardless of the polymer used, although the onset of crystallisation time varies from zero to 16 weeks. Additionally, differences were observed between the two methods, spray-dried formulations seem to be more stable for PVP-VA and HPMCAS while the opposite is true for SOL formulations. 


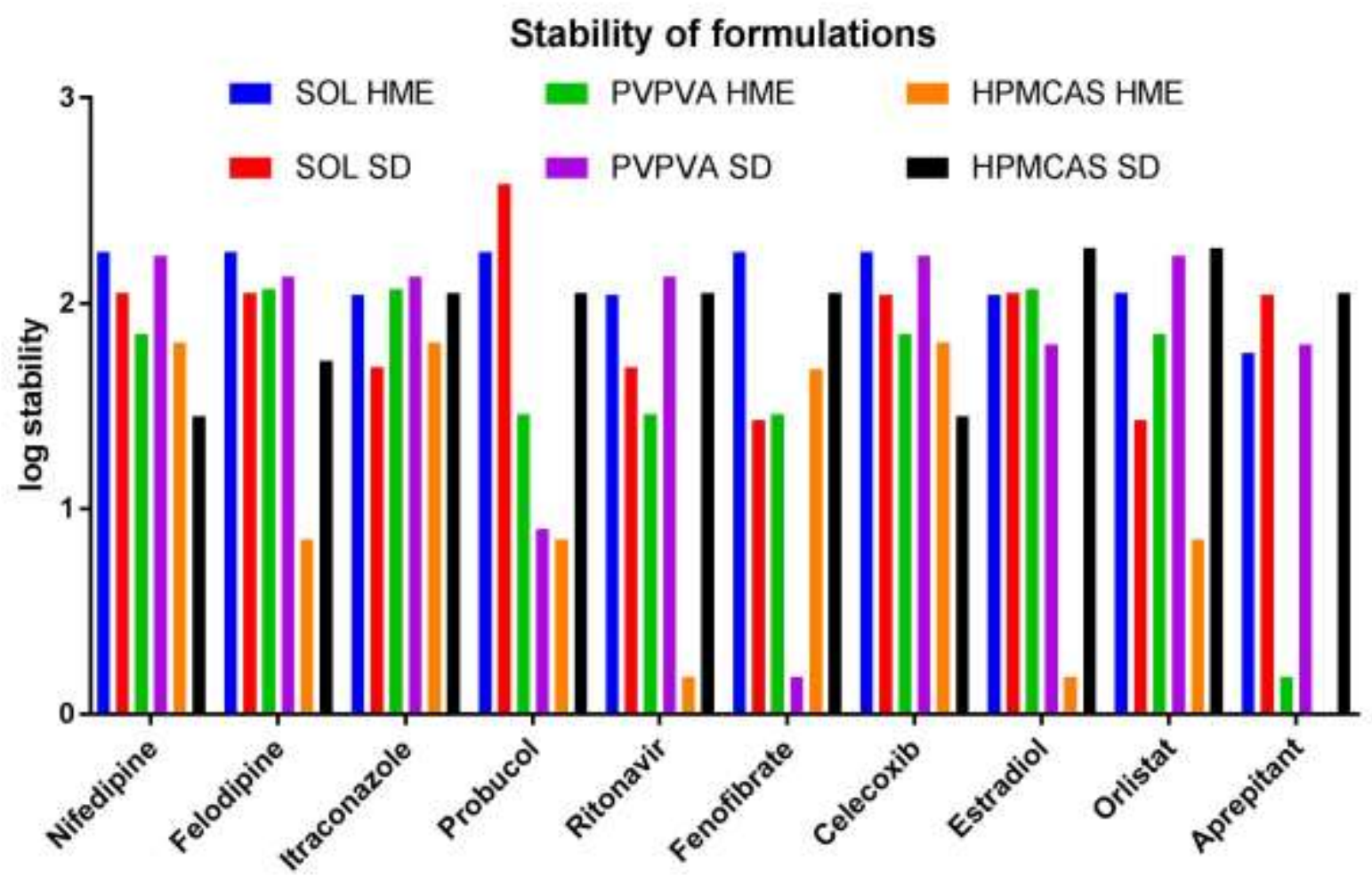

FIGURE 6 shows measured stability of the solid dispersions (last time point were no crystals were observed plotted as $\log ($ Stability).

\section{MLR MODELS}

Six statistical MLR models were constructed, one for each combination of polymer and manufacturing method. Even though each modelling process started with the same predicted, calculated, or measured parameters, none of the models were identical, each indicating that different parameters were influential in the stability of that particular polymer dispersion and manufacturing method. This provides a direct indication of why it has been difficult to find agreement among different studies on the most important parameters to consider when seeking the most stable solid dispersion formulation and production method.

Due to the relatively limited dataset (although very large compared to previous studies), the number of independent physicochemical parameters in each model was limited to three. Models containing one and two parameters were also built as well as models containing pooled datasets. Additionally, there is a greater risk of overfitting when using small data sets which is why leave-one-out validation was used to explore the general features of the data. The first step was determining which three parameters had the strongest correlation with the stability of the formulations. 
Table 3 an overview of the abbreviations used for the different polymer - manufacturing method combinations

\begin{tabular}{c|l} 
Abbreviation & Formulation \\
\hline$S h$ & SOL HME \\
$S s$ & SOL spray-dried \\
$P h$ & PVP-VA HME \\
$P s$ & PVP-VA spray-dried \\
$H h$ & HPMCAS HME \\
$H s$ & HPMCAS spray-dried
\end{tabular}

Once the three most influential parameters had been chosen, a MLR model was derived using the stability of the ten drugs and the parameters. The models, adjusted $R^{2}\left(R^{2}\right.$ adj $)$ and mean square error are listed in Table 4. The Sh stability model shows a negative correlation with the number of $\mathrm{H}$-bond acceptors and the number of chiral atoms, and a positive correlation with topological polar surface area. If number of $\mathrm{F}$ atoms was included in the model the adjusted $\mathrm{R}^{2}$ was 0.918 and the MSE was 0.08 , the model was then $\log$ (stability) $=2.675-0.04 \mathrm{nF}-0.132$ nAliphRing - 0.001 MW. Ss formulation stability is positively correlated with measured glass transition temperature $\left(T_{g \text {,meas }}\right)$ and molecular polarizability while negatively correlated with the number of $\mathrm{N}$ atoms in the drug. Stability of $\mathrm{Ph}$ shows a positive correlation with predicted glass transition temperature ( $\left.T_{g, p r e d}\right)$ and a negative correlation with number of H-bond donors and the melting point of the API. The nitrogen count and $T_{g \text {,pred }}$ are positively correlated with stability of Ps while the number of aromatic atoms shows a negative correlation. The Hh stability shows negative correlation with number of HBD and lipophilicity while sulphur count is positively correlated. Both ring count and number of rotatable bonds is positively correlated with the stability of HPMCAS spray-dried but the number of nitrogen atoms is negatively correlated. The optimal models have $\mathrm{R}^{2}$ adj values between 0.798 and 0.895 .

The MSE was calculated from a leave-one-out validation of each model. In a leave-one-out cross validation (LOOCV) one of the data points (formulations) is excluded from the dataset used to build the model. The same procedure is used as with the whole dataset and the parameters are selected before the model is built. For example, a model was built leaving out the nifedpine data. The new model is then used to predict the stability of the drug that was excluded, nifedipine. This is repeated ten times, each time excluding one API and predicting the stability of that API. The MSE of these predictions was then calculated and it gives an indication of the predictability of the models. The comparison of predicted and measured stability from each LOOCV can be seen in Figure 7. Substantial difference in MSE can be seen between the models (see both Figure 7 and Table 4), the lowest MSE is in the Sh model, 0.08, while the highest is seen in the Hh model, 3.481.

Models were also made using only one and two parameters (Table 4), these had lower $\mathrm{R}^{2}$ adj (0.231-0.659) and (0.621-0.781) respectively but in most cases lower MSE (0.029-7.445) and (0.068-2.619). Attempts to build models using pooled data were unsuccessful, with $\mathrm{R}^{2}$ adj ranging from -0.1 to 0.33 . These included six pooled dataset, one including all data, one for 
each method - containing data from all polymers and one for each polymer which included both methods.

TABLE 4. Summary of models, including adjusted $\mathrm{R}^{2}$ and MSE values. The following abbreviations are used, nCov - number of covariants, $\mathrm{R}_{\text {adj }}^{2}$ - adjusted $\mathrm{R}^{2}$, MSE - mean square error, $\operatorname{logStab}$ - log Stability, tPSA - topological polar surface area atoms, $\mathrm{HBA}-\mathrm{H}, \mathrm{mp}-$ melting point, $\mathrm{nF}$ - number of $\mathrm{F}$ atoms, $\mathrm{nS}$ - number of $\mathrm{S}$ atoms, $\mathrm{nN}$ - number of $\mathrm{N}$ atoms, $\mathrm{HBD}-$ number of $\mathrm{H}$ bond donors, $\mathrm{nRot}$ - number of rotatable bonds, molPol - molecular polarizability, $T_{g p r e d}$ - predicted $T_{g}$ using the Gordon-Taylor equation, $T_{\text {gmeas }}$ - measured $T_{g}$, nRing - number of rings, nAr - number of aromatic atoms, nHet - number of heteroatoms, $\mathrm{sp}^{3}-$ number of $\mathrm{sp}^{3}$ carbon atoms.

\begin{tabular}{|c|c|c|c|c|}
\hline Name & $\mathrm{nCov}$ & Model & $\mathrm{R}_{\text {adj }}^{2}$ & MSE \\
\hline Sh & 3 & $\operatorname{logStab}=2.307+0.003$ tPSA -0.055 nChir $-0.082 \mathrm{HBA}$ & 0.891 & 0.08 \\
\hline Ss & 3 & logStab $=-3.864+0.081 T_{g, \text { meas }}-0.163 n \mathrm{~N}+0.015 \mathrm{molPol}$ & 0.895 & 0.265 \\
\hline $\mathrm{Ph}$ & 3 & $\operatorname{logStab}=-11.2106-0.2355 H B D-0.0112 \mathrm{mp}+0.1536 T_{\text {g,pred }}$ & 0.815 & 1.021 \\
\hline Ps & 3 & $\operatorname{logStab}=-5.252+0.079 T_{g, p r e d}+0.390 n N-0.119 n A r$ & 0.798 & 2.113 \\
\hline $\mathrm{Hh}$ & 3 & $\log S t a b=2.472-0.849 H B D-0.124 C \log P+0.771 n S$ & 0.827 & 3.481 \\
\hline Hs & 3 & logStab $=0.953+0.055 n R o t+0.331 n$ Ring $-0.216 n N$ & 0.870 & 0.781 \\
\hline Sh & 2 & $\operatorname{logStab}=1.988+0.078 n S-0.042 p K a_{b}$ & 0.781 & 0.068 \\
\hline Ss & 2 & logStab $=-1.829-0.059 \mathrm{nHet}+0.063 T_{g, \text { meas }}$ & 0.647 & 0.308 \\
\hline $\mathrm{Ph}$ & 2 & $\operatorname{logStab}=-0.605-0.232 n F+0.025 T_{g, \text { meas }}$ & 0.651 & 1.517 \\
\hline Ps & 2 & $\operatorname{logStab}=2.116-0.104 n A r-0.389 n N$ & 0.621 & 1.052 \\
\hline $\mathrm{Hh}$ & 2 & $\operatorname{logStab}=1.754-0.711 H B D+0.480 n S$ & 0.688 & 2.619 \\
\hline $\mathrm{Hs}$ & 2 & $\log S t a b=1.415+0.060 \log P+0.102 n C h i r$ & 0.700 & 0.276 \\
\hline Sh & 1 & $\operatorname{logStab}=2.033-0.042 p K a_{b}$ & 0.659 & 0.029 \\
\hline Ss & 1 & $\operatorname{logStab}=-0.667+0.038 T_{g, \text { meas }}$ & 0.272 & 0.392 \\
\hline $\mathrm{Ph}$ & 1 & $\operatorname{logStab}=1.831-0.199 n F$ & 0.605 & 0.394 \\
\hline Ps & 1 & $\log S t a b=0.839+0.012 t P S A$ & 0.231 & 7.445 \\
\hline $\mathrm{Hh}$ & 1 & $\operatorname{logStab}=1.669-0.479 H B D$ & 0.534 & 0.520 \\
\hline $\mathrm{Hs}$ & 1 & $\log S t a b=1.599+0.030 s p^{3}$ & 0.483 & 0.149 \\
\hline
\end{tabular}


Soluplus HME

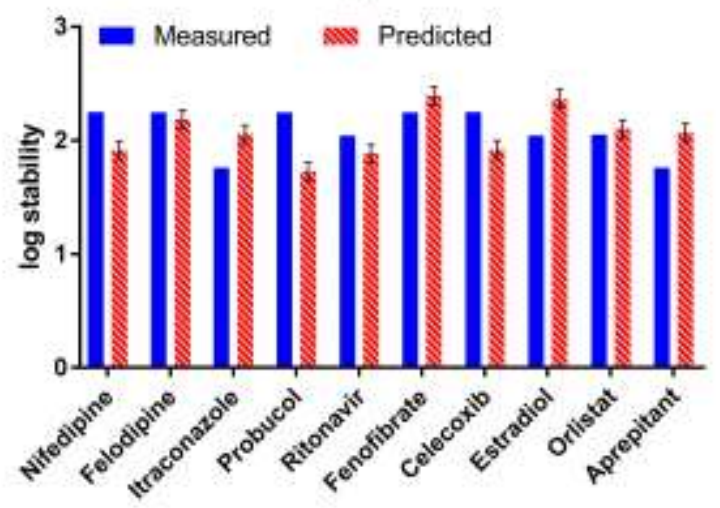

PVPVA HME

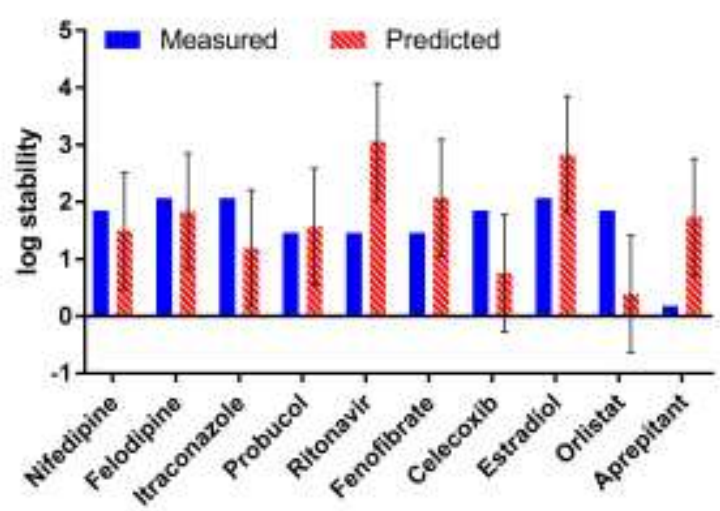

HPMCAS HME

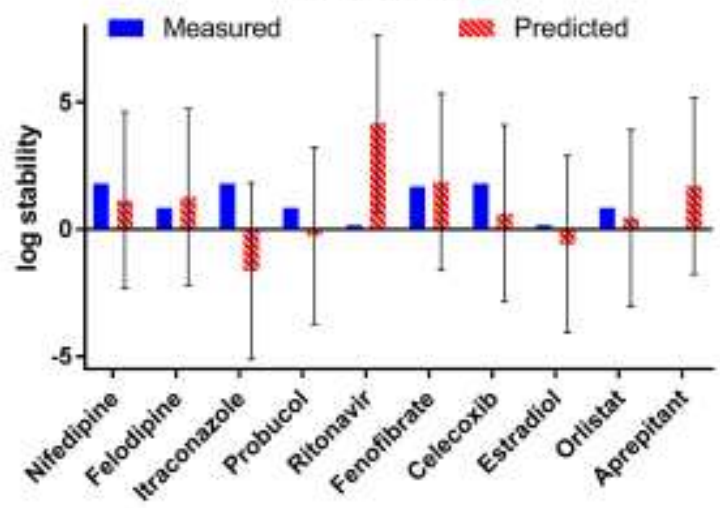

Soluplus Spray-dried

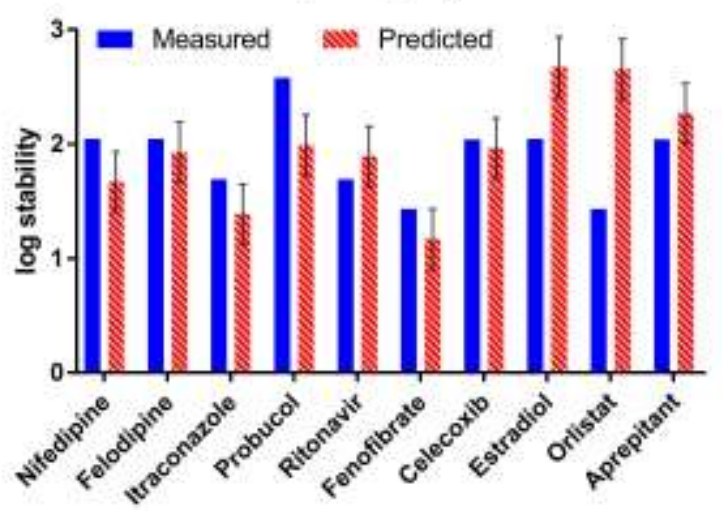

PVPVA spray-dried

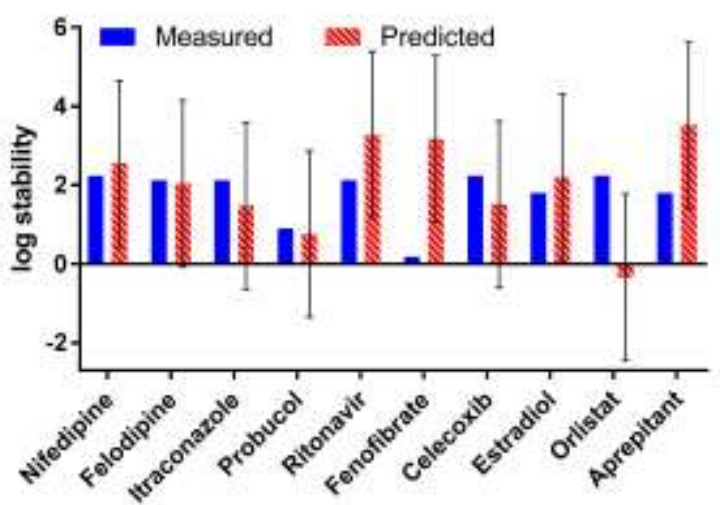

HPMCAS spray-dried

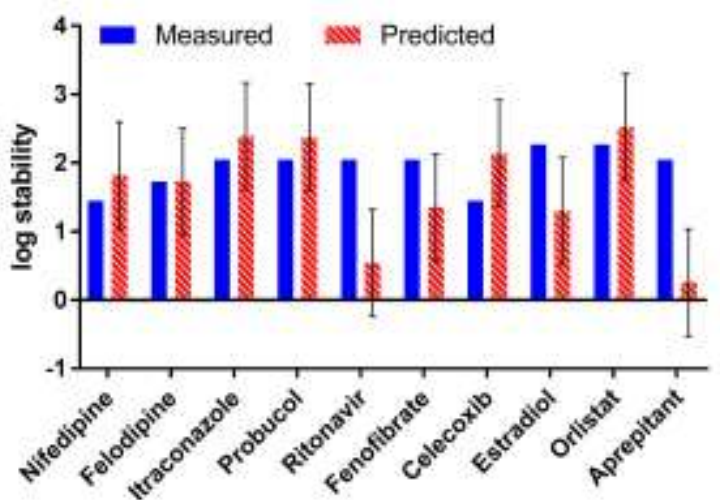

FIGURE 7 Leave-one-out cross validation of each of the six models. The error bars on the predicted stability is the MSE of each model.

A further external validation was performed. A recent study compared the stability of three solid dispersion formulations of lapatinib (structure Table 5), namely SOL, PVP-VA and 
HPMCAS. These were spray-dried at $40 \% \mathrm{w} / \mathrm{w}$ API and stored at $40{ }^{\circ} \mathrm{C} / 75 \% \mathrm{RH}$. It was found that the formulation containing HPMCAS was the most stable by far, staying amorphous for the six months tested while both PVP-VA and SOL formulations were crystalline by one month ${ }^{36}$. Using our Ss, Ps and Hs models, the stability of lapatinib formulations was predicted in each polymer. The models ranked the stability correctly both using two- and three-parameter models. For the three-parameter models the stability was less than one month for SOL and PVP-VA and more than six months for HPMCAS, reproducing perfectly the measured stability (Table 5)

Table 5. Measured ${ }^{36}$ and predicted stability (using three covariant models) of lapatinib formulations. The molecular structure of lapatinib (right).

\begin{tabular}{|l|l|l|l|}
\hline & SOL & PVP-VA & HPMCAS \\
\hline Measured & $<1$ month & $<1$ month & $>6$ months \\
\hline Predicted & 19 days & 0 days & 220 days \\
\hline
\end{tabular}

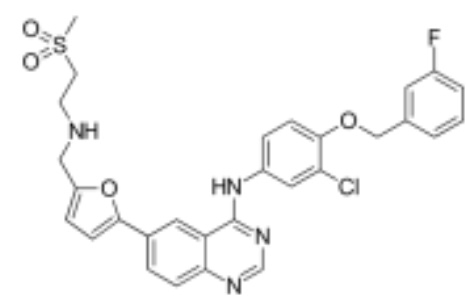

\section{DISCUSSION}

Most studies considering the stability of solid dispersions solely rely on DSC and XRPD for detecting crystals, not PLM ${ }^{7,37-40}$. However it has been observed in this study that specifically at low drug loading these two methods lack the sensitivity to detect onset of crystallinity accurately. Here PLM has therefore been used as a primary method of detection as it is known to be a sensitive and rapid method to detect small amounts of crystalline material in amorphous matrices $^{41-43}$.

\section{STABILISING POLYMER}

For some drugs there is a clear difference in stability between polymers while for others there is minimal difference. For instance probucol was very stable in both formulations containing SOL. No crystals were observed up to six months in HME and it was still amorphous after one year in spray-dried formulations. Probucol was the only drug that stayed amorphous in spraydried SOL for more than four months and the only formulation to stay amorphous for the whole year tested. However for PVP-VA it was one of the first APIs to show signs of crystallisation in both methods, 4 and 8 weeks, respectively for spray-dried and HME. In HPMCAS the crystallisation onset was determined to be 4 weeks for HME and 24 weeks for spray-dried. When considering the physicochemical properties of probucol, ClogP stands out as extreme. Probucol has a ClogP of 10, which is the highest of the APIs used in this study. It is possible that this attribute is connected with this difference in stability, the PEG and PCL parts of SOL may better stabilise this highly lipophilic molecule compared to the more hydrophilic PVPVA, and the amphiphilic HPMCAS ${ }^{44}$. Other drugs such as fenofibrate started crystallising at 
the same time for all three polymers, at 8 weeks for HME HPMCAS and PVP-VA and spraydried SOL.

When comparing the stability of spray-dried solid dispersions it is interesting to note that for each of the polymers, two APIs had shown signs of crystallisation by two months. However out of the six crystallised formulations only two contained the same drug. For HPMCAS it was celecoxib and nifedipine; probucol and fenofibrate for PVP-VA; and orlistat and fenofibrate for SOL. It can therefore be assumed that this difference is due to the stabilising effect of the polymer or a lack thereof. Even the least stable drugs behave quite differently depending on the polymers. This would indicate that relative stability is not solely due to the method used or the inherent instability of the API, but how well the polymer can influence the physical stability of the dispersed drug.

Using a polymer with a high $T_{g}$ will increase the $T_{g}$ of its solid dispersions as the API $T_{g}$ values are generally quite low (here ranging from $-45^{\circ} \mathrm{C}$ to $85^{\circ} \mathrm{C}$ ). Solid dispersions with high $T_{g}$ values are generally thought to be more stable than ones with a lower $T_{g}{ }^{16}$. Although some studies have found a strong correlation between increasing polymer $T_{g}$ and decreasing crystallisation tendency, others have found no such connection or even shown an opposite trend ${ }^{28-31}$. In this study the three polymers used have a wide range of physicochemical properties, such as $T_{g}$, SOL $70{ }^{\circ} \mathrm{C}$, PVP-VA $103{ }^{\circ} \mathrm{C}$ and HPMCAS $120{ }^{\circ} \mathrm{C}{ }^{45-47}$. The results show that generally $\mathrm{SOL}$ is the most stabilising polymer in HME formulations despite having the lowest glass transition temperature. The same does not apply to the spray-dried formulations where PVP-VA and HPMCAS show better crystallisation inhibition properties. However, PVP-VA and HPMCAS were spray-dried at a temperature below their $T_{g}$ while for SOL this was above its $T_{g}$. Whether this affected stability is unclear. It is clear, however, that there are more factors that contribute to stability than just the polymer or solid dispersion $T_{g}$.

\section{STABILISING INTERACTIONS}

Generally there are four different types of specific interactions that can occur between an API and a polymer, in order of decreasing bond strengths: ionic bonds > hydrogen bonds > dipoledipole interactions $>$ van der Waals interactions. Ionic bonds are strong attractions between two oppositely charged molecules. A hydrogen bond (H-bond) is an electrostatic interaction between a hydrogen and an electronegative atom $(\mathrm{O}, \mathrm{N}$ or $\mathrm{F})$, these can occur both between two identical molecules (API-API) and different molecules (polymer-API). A dipole-dipole interaction can occur between two polar molecules, when they line up so that the positive part of one molecule is opposite the negative one of the other molecule. The weakest of the interactions is the van der Waals interactions, which are induced dipolar interactions that can occur between any functional groups. Specific interactions between drug and polymer can impact the physical stability of solid dispersions. For instance H-bonds between polymer and drug can hinder crystallisation by reducing the amount of drug in the conformation needed for crystallisation. H-bonding can also occur between APIs, which can facilitate crystallisation. However, if there are more or stronger H-bonds available between API and polymer, or amorphous API-API than between crystalline API-API this can impede crystallisation $16,44,48$. 
In a study of solid dispersions containing vitamin $\mathrm{C}$ and several polymers the influence of $\mathrm{H}$ bonding between polymer and drug on physical stability was considered. As well as H-bonding, the effect of polymer hygroscopicity and $T_{g}$ were examined. It was found that the formation of strong $\mathrm{H}$-bonds between the polymer and drug in dispersions was the most important factor inhibiting crystallisation of vitamin $\mathrm{C}$. The hygroscopicity of the polymer played some part in stability, especially when the solid dispersions were stored at a high RH. No correlation could be found between polymer $T_{g}$ and physical stability of solid dispersions ${ }^{28}$. In a similar study performed by Van den Mooter et al. it was observed that in absence of any polymer-drug interaction, polymer $T_{g}$ was an important stabilising factor ${ }^{30}$.

Functional groups that can act as H-bond acceptors or donors are present in all three polymers. H-bond donors include alcohol and carboxylic acid both present in HPMCAS. Amide (PVPVA, SOL), esters (SOL, PVP-VA and HPMCAS) and ethers (HPMCAS, SOL) can all act as H-bond acceptors ${ }^{42}$. Thus only HPMCAS has H-bond donors while all polymers have H-bond acceptors present. In Table 2 the number of H-bond donors and acceptors are listed for each of the drugs. It can be observed that these numbers vary greatly between the different drugs, Hbond acceptors range from two to nine while $\mathrm{H}$-bond donors range from zero to four. If the stability of solid dispersions was mostly dependent on the ability of the drug and polymer to make specific bonds such as $\mathrm{H}$-bonds it would be expected that the drugs with large number of $\mathrm{H}$-bond acceptors would be more stable in HPMCAS than PVP-VA and SOL while drugs with no H-bond donors would not be sufficiently stabilised by PVP-VA and SOL as no interaction could be made. Itraconazole, which has the greatest number of $\mathrm{H}$-bond acceptors, first showed signs of crystallisation at 16 and 24 weeks, respectively for HME and spray-dried HPMCAS solid dispersions. However, both aprepitant and ritonavir were very unstable in HME HPMCAS but both have six H-bond acceptors. Fenofibrate, which has no H-bond donors, was the least stable spray-dried formulations for both SOL and PVP-VA but the HME SOL was stable through the whole six months of testing. It is therefore clear that there are further properties that contribute to the stability than just the ability of drug and polymer to interact, even though that is a probably important factor.

Water, once absorbed into the formulation, can disrupt any H-bonding between the drug and polymer. Furthermore water can act as a plasticizing agent and increase molecular mobility 49,50. Thus even if a solid dispersion formulation started out stable with abundance of stabilising $\mathrm{H}$-bonds, at accelerated conditions these might be removed by water and thus cause instability and crystallisation. In most cases for SOL and PVP-VA, crystallisation was not observed while the spray-dried formulations were still a dispersible powder but only once they had transformed into a solid glass, which indicates the presence of water. PVP-VA is a very hydrophilic polymer and thus quite hygroscopic, absorbing $10 \%$ water at $50 \%$ RH. Relative to PVP-VA, HPMCAS and SOL are less hygroscopic, taking up 6-7\% water at $75 \% \mathrm{RH}$ and $10 \%$ water at $70 \% \mathrm{RH}$, respectively ${ }^{51}$. The effect differences in hygroscopy have on the stability of the formulations can be seen clearly in the case of the spray-dried formulations above. Especially when comparing formulations with PVP-VA and HPMCAS. PVP-VA spray-dried formulations form a glass after a while when exposed to very high humidity, $75 \% \mathrm{RH}$. The powder seems to melt 
and/or dissolve into a glass and simultaneously crystals start forming. This transformation of powder into glass did not occur for the HPMCAS spray-dried formulations, even after one year at high humidity. It was deduced that this transformation was mainly due to the hygroscopy of the PVP-VA and SOL polymer, as it was not seen at less humid conditions (data not shown). As water negatively affects the stability of the dispersion, this transformation can be regarded as a step towards crystallisation. Despite the transformation of spray-dried formulations due to uptake of water for PVP-VA formulations they were still overall, more stable than the corresponding HME formulations.

\section{PROCESSING METHOD}

Janssens and coworkers studied the influence of processing method on the stability of an itraconazole - Eudragit (acrylic polymers often used in solid dispersion formulations) solid dispersion ${ }^{52}$. Comparing solid dispersions produced with three different methods, spraydrying, film-casting, and hot melt extrusion. The maximum drug loading that could be stabilised by Eudragit was $27.5 \%$ for spray-drying and $15 \%$ for each of the other two methods. This shows that the processing method can have significant effects on the stabilisation of solid dispersions ${ }^{52}$.

A comparison of HME and spray-drying in regard to interaction, drug distribution and homogeneity indicated a lower achievable drug loading by HME than spray-drying. However, HME formulations were more homogenous, stable and showed stronger interactions than the spray-dried examples ${ }^{53,54}$.

Some effects on stability are due to the processing method. In some cases a reason might be quite clear, such as aprepitant being the least stable drug in HME formulations. Aprepitant has a very high melting point, $255^{\circ} \mathrm{C}$ and was extruded at a low temperature $\left(160^{\circ} \mathrm{C}\right)$. Other effects of the processing method are not as clear. Only in four cases was the time of crystallisation onset the same for the two methods in two similar formulations, these are SOL-itraconazole, SOL-estradiol, PVP-VA -itraconazole and PVP-VA - felodipine. In all other cases the onset of crystallisation time was differed between the two methods. The largest difference was observed for SOL-fenofibrate, where the spray-dried formulation showed signs of crystals after eight weeks while no crystals were observed in the HME until after one year.

When comparing the two processing methods it can be observed that overall, for SOL, HME seems to produce more stable solid dispersions than spray-dried, while the opposite appears to be the case for both PVP-VA, and HPMCAS. This indicates that the processing method has a profound effect on the solid dispersions stability. It is unlikely that specific bonds would only be present using one method not the other, therefore relative stability must be directly linked with attributes of the methods. These might include, for example, residual solvents that can destabilise the solid dispersion, in case of spray-dried formulations. Different compounds can retain varying amounts of solvent which might in some cases be responsible for the observed difference in stability. 


\section{MLR MODELS}

All six models (Table 4) contain three physicochemical parameters, but no two models contain the same three, showing the complexity of the stability of solid dispersions. However some general rules can be deducted from the six models. The following are correlated with increased stability of the solid dispersion.

1. Increased $T_{g}$ of the dispersion (measured or predicted)

2. Increased molecular flexibility of the API

3. Decreased number of H-bond donors (or Fluorine / Nitrogen atoms)

The first rule is in good agreement with previous studies. As stated above many studies have considered the correlation between $T_{g}$ and solid dispersion stability ${ }^{55}$. This often relates to choosing a high $T_{g}$ polymer in order to obtain a solid dispersion with a high $T_{g}$. Moreover, storing the solid dispersion $50^{\circ} \mathrm{C}$ below the $T_{g}$ of the solid dispersion reduces the molecular mobility to a minimum, thus increasing the stability (reviewed in ${ }^{56}$ ). Thus, for storing the solid dispersion at $40^{\circ} \mathrm{C}$, a solid dispersion with at least a $T_{g}$ of $90{ }^{\circ} \mathrm{C}$ would be preferable. Here, 60 $\%$ of the solid dispersions tested had a $T_{g}$ at least $50{ }^{\circ} \mathrm{C}$ above the storage temperature.

The second rule indicates that the more flexible API molecules are, the less likely they are to adopt the correct conformation for crystallisation. In the models the parameters relating to flexibility are aromatic atom count, rotatable bonds, chiral atoms and ring count. The parameters hindering flexibility, aromatic atom count and chiral atoms, were negatively correlated with stability while the opposite was observed for rotatable bonds, which increases flexibility. Ring count was positively correlated although it would be expected to be negative, however, this parameter only occurred in the model with the lowest $\mathrm{R}^{2}$ adj. Aromatic rings can interact strongly through van der Waals interactions and $\pi-\pi$ stacking, symmetry drives interactions and intermolecular bonding, while ring structures have been showed to cause dense packing structure in crystals, all of which can drive crystallisation ${ }^{18}$.

A predictive model using a partial least squares projection to latent structure discriminant analysis (PLS DA) was built based on experimentally determined glass forming ability of 16 drugs. The model showed a negative correlation between glass forming ability and number of benzene rings, but a positive correlation to branching of the carbon skeleton, molecular symmetry and distribution of electronegative atoms in the API ${ }^{57}$. Another study found that APIs with high molecular weight and flexible structures were more likely to be glass formers than small rigid molecules ${ }^{58}$. Several models using calculated, predicted and measured parameters have been built to predict the amorphous stability of APIs. In models containing rotatable bonds these were positively correlated with stability whilst aliphatic rings were negatively correlated ${ }^{23}$. These studies only examined the API alone and not in a solid dispersion as here, moreover two of them only look at glass-forming ability, not stability. However, they show that flexibility and structural complexity of the API is strongly connected with its crystallisation tendency, which was highlighted by four flexibility/complexity (nChir) parameters in the models above. It can therefore be concluded that in regards to flexibility of the API, the same applies to glass forming ability of API on its own and the stability of the amorphous form in a solid dispersion. 
The first two rules are intuitively expected whereas the third one is less so. H-bonds are an important factor to stabilise solid dispersion as discussed before. Several studies have found hydrogen bonding to be important for the glass forming ability or stability of glasses ${ }^{22,23,59}$. Furthermore, a positive correlation was found between well-distributed electronegative atoms and glass forming ability, which may be due to the H-bonding ability of such atoms ${ }^{18,57}$. The atomic mass-weighted third-order $\mathrm{R}$ autocorrelation index $(\mathrm{R} 3 \mathrm{~m})$ molecular descriptor has been found to be the most significant predictor of miscibility in PVP-VA solid dispersion. This was based on experimental data from 12 APIs. The R3m is associated with electronegative atoms and their positions in the molecule ${ }^{60}$. None of these studies considered the stability of solid dispersions in the context of H-bonding. Here, however, H-bond donors and acceptors, as well as number of nitrogen atoms are shown to be negatively correlated with stability, in all cases but for $\mathrm{N}$ count in the Ps model. For the three polymers studied here only HPMCAS has any H-bond donors, but all have H-bond acceptors. However, even if there are H-bond acceptors available in the carrier polymer that does not necessarily mean that API-polymer Hbonds will actually be formed. Instead these H-bond donors/acceptors in the APIs could lead to API-API bonding or API-water H-bonding, both of which lead to decreased stability and more rapid crystallisation.

Our proposed rules are not intended to be absolute but to suggest guidelines, and, due to the complexity of these systems, exceptions must be expected. E.g., even though increased $T_{\mathrm{g}}$ is correlated with increased stability of SOL HME, solid dispersions were the most stable of the HME formulations overall, despite SOL having the lowest $T_{\mathrm{g}}$ out of the three polymers. Similar examples can be found for the flexibility. If the two extremes are compared, estradiol is the most rigid API in the study with three aliphatic rings, one aromatic ring and no rotatable bonds. Orlistat is the other extreme with only one aliphatic ring, no aromatic rings and 23 rotatable bonds. If solid dispersion stability was only dependent on API flexibility orlistat would be expected to be much more stable than estradiol in all formulations. However, this is not the case, orlistat is more stable in two formulations PVP-VA spray-dried (52weeks vs 26 weeks) and HPMCAS HME (4 weeks vs 1 week). In two instances the stability is the same for the two drugs in comparable formulations: SOL HME and HPMCAS spray-dried, while in the latter two formulations estradiol is more stable than orlistat.

The MSE ranges from minimal in Sh model to very large in the PVP-VA models in particular. If the predicted values are compared to the measured ones they are overall mostly in good agreement. However, especially for the PVP-VA and Hh models there are a few APIs that are very poorly predicted, such as ritonavir in both $\mathrm{Ph}$ and $\mathrm{Hh}$ or fenofibrate in Ps. These, during the LOOCV process, were predicted using models that varied from the main models. The LOOCV models for these drugs used parameters for which the APIs had extreme values, differing greatly from the rest, such as for ritonavir both $\mathrm{Ph}$ and $\mathrm{Hh}$ models contained tPSA which in both cases ritonavir has the highest value by far or 145 compared to most other well under 100. This difference in chosen models in LOOCV is mostly due to the relatively small dataset, with more data the models are more robust and less likely to be as affected by leaving out a single value. 
The purpose of these models is to indicate which combination of processing method and polymer carrier is most likely to give a stable solid dispersion. Thus the models are used to rank the stability of the formulations so that the best combination can be found. Figure 8 shows the ranking of formulation combinations. 

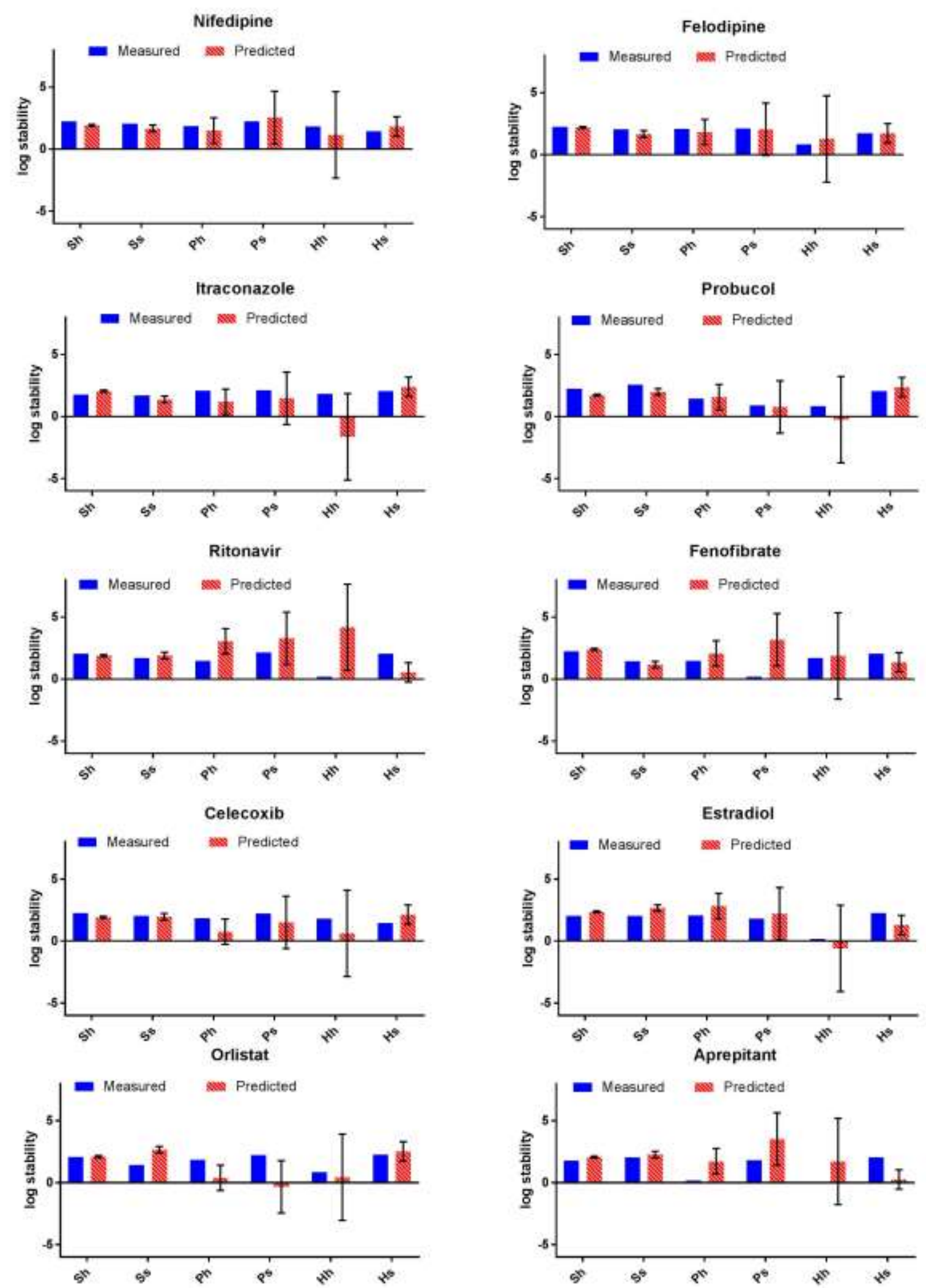

FIGURE 8 Ranking of formulation combinations each API and polymer-manufacturing combination. The error bars show the MSE of each model. The predicted ranking is in good agreement with the measured stability. The following abbreviations are used: SH - SOL HME, SS - SOL spray-dried, PH - PVP-VA HME, PS - PVP-VA spray-dried, HH - HPMCAS HME and HS - HPMCAS spray-dried. 
When comparing the models using one, two and three covariates the following can be observed, as expected the $\mathrm{R}^{2}$ adj falls when number of covariates is decreased. However when looking at the MSE this decreases in four cases out of six. The lower MSE might suggest that the higher number of covariates is over-fitting the model. In order to test the models against over-fitting an external validation is needed.

The external validation shows that the models are nevertheless able to correctly predict the ranking of formulations containing a novel drug. Moreover the predicted stability was in very good agreement with the measured stability, thus showing that despite a relatively small dataset, in terms of statistics, the models can be applied on real data to give a true ranking of formulations, which is promising as a support tool in solid dispersion formulation development.

\section{CONCLUSIONS}

60 solid dispersions were produced successfully using ten APIs, three polymers and two processing methods. An accelerated stability study has shown significant difference in time to crystallisation dependent on both choice of polymers and processing method. This extensive experimental dataset provides new insights into the complexity of the stability of solid dispersion systems. There are, as previously described, many factors that influence their stability. However, there are some conclusions that can be drawn, such as HPMCAS and PVPVA are more likely to give a stable formulation using spray-drying, while HME seems to be more favourable for SOL solid dispersion formulations.

Six predictive statistical models were built, which show the potential of using such models in formulation development. Furthermore, none of the models are identical which indicates the complexity of these systems. Three rules that could guide formulation development can be deduced. Increased stability is correlated with increased $T_{\mathrm{g}}$ of solid dispersion, as are decreased number of H-bond donors and increased molecular flexibility of the API. The LOOCV showed good predictability which was confirmed in an external validation of three formulations.

Future studies are planned to add data to the models to further increase their robustness and possibly to use as an external validation set.

\section{ACKNOWLEDGMENTS}

This work was supported by the Engineering and Physical Sciences Research Council [grant number EP/I01375X/1] via a PhD sponsorship for Gudrun A. Fridgeirsdottir and by Juniper Pharmaceutical Services at the Centre for Doctoral Targeted Therapeutics and Drug Formulation at the University of Nottingham. 


\section{REFERENCES}

(1) Di, L.; Kerns, E.; Carter, G. Drug-like Property Concepts in Pharmaceutical Design. Curr. Pharm. Des. 2009, 15 (19), 2184-2194.

(2) Di, L.; Fish, P. V; Mano, T. Bridging Solubility between Drug Discovery and Development. Drug Discov. Today 2012, 17 (9-10), 486-495.

(3) Dai, W. G.; Pollock-Dove, C.; Dong, L. C.; Li, S. Advanced Screening Assays to Rapidly Identify Solubility-Enhancing Formulations: High-Throughput, Miniaturization and Automation. Adv. Drug Deliv. Rev. 2008, 60, 657-672.

(4) Williams, H. D.; Trevaskis, N. L.; Charman, S. a; Shanker, R. M.; Charman, W. N.; Pouton, C. W.; Porter, C. J. H. Strategies to Address Low Drug Solubility in Discovery and Development. Pharmacol. Rev. 2013, 65 (1), 315-499.

(5) Vasconcelos, T.; Sarmento, B.; Costa, P. Solid Dispersions as Strategy to Improve Oral Bioavailability of Poor Water Soluble Drugs. Drug Discov. Today 2007, 12 (23-24), 1068-1075.

(6) Karavas, E.; Georgarakis, M.; Docoslis, A.; Bikiaris, D. Combining SEM, TEM, and Micro-Raman Techniques to Differentiate between the Amorphous Molecular Level Dispersions and Nanodispersions of a Poorly Water-Soluble Drug within a Polymer Matrix. Int. J. Pharm. 2007, 340 (1-2), 76-83.

(7) Vo, C. L.-N.; Park, C.; Lee, B.-J. Current Trends and Future Perspectives of Solid Dispersions Containing Poorly Water-Soluble Drugs. Eur. J. Pharm. Biopharm. 2013, 85 (3 Pt B), 799-813.

(8) Pereira, J. M.; Mejia-Ariza, R.; Ilevbare, G. a; McGettigan, H. E.; Sriranganathan, N.; Taylor, L. S.; Davis, R. M.; Edgar, K. J. Interplay of Degradation, Dissolution and Stabilization of Clarithromycin and Its Amorphous Solid Dispersions. Mol. Pharm. 2013, 10 (12), 4640-4653.

(9) Kawabata, Y.; Wada, K.; Nakatani, M.; Yamada, S.; Onoue, S. Formulation Design for Poorly Water-Soluble Drugs Based on Biopharmaceutics Classification System: Basic Approaches and Practical Applications. Int. J. Pharm. 2011, 420 (1), 1-10.

(10) Saboo, S.; Taylor, L. S. Water-Induced Phase Separation of Miconazole-Poly (Vinylpyrrolidone-Co-Vinyl Acetate) Amorphous Solid Dispersions: Insights with Confocal Fluorescence Microscopy. Int. J. Pharm. 2017, 529 (1-2), 654-666.

(11) Singh, A.; Van den Mooter, G. Spray Drying Formulation of Amorphous Solid Dispersions. Adv. Drug Deliv. Rev. 2016, 100, 27-50.

(12) Crowley, K.; Gryczke. Hot Melt Extrusion of Amorphous Solid Dispersions. In Pharmaceutical amorphous solid dispersions; Newman, Ed.; John Wiley \& Sons, Inc.: Hoboken, New Jersey, 2015; pp 341-363.

(13) Li, B.; Konecke, S.; Wegiel, L. a; Taylor, L. S.; Edgar, K. J. Both Solubility and Chemical Stability of Curcumin Are Enhanced by Solid Dispersion in Cellulose Derivative Matrices. Carbohydr. Polym. 2013, 98 (1), 1108-1116.

(14) Leuner, C.; Dressman, J. Improving Drug Solubility for Oral Delivery Using Solid 
Dispersions. Eur. J. Pharm. Biopharm. 2000, 50 (1), 47-60.

(15) EMA. European Medical Agency http://www.ema.europa.eu/ema/ (accessed Jun 15, 2015).

(16) Janssens, S.; Mooter, G. Van Den. Review : Physical Chemistry of Solid Dispersions. J. Pharm. Pharmacol. 2009, 61, 1571-1586.

(17) Kuentz, M.; Holm, R.; Elder, D. P. Methodology of Oral Formulation Selection in the Pharmaceutical Industry. Eur. J. Pharm. Sci. 2016, 87, 136-163.

(18) Bergström, C. A. S.; Charman, W. N.; Porter, C. J. H. Computational Prediction of Formulation Strategies for beyond-Rule-of-5 Compounds. Advanced Drug Delivery Reviews. The Authors 2016, pp 6-21.

(19) Fridgeirsdottir, G. A.; Harris, R.; Fischer, P. M.; Roberts, C. J. Support Tools in Formulation Development for Poorly Soluble Drugs. J. Pharm. Sci. 2016, 105 (8), 2260-2269.

(20) DeBoyace, K.; Wildfong, P. The Application of Modeling and Prediction to the Formation and Stability of Amorphous Solid Dispersions. J. Pharm. Sci. 2017, 1-18.

(21) Bergström, C. A. S.; Charman, W. N.; Porter, C. J. H. Computational Prediction of Formulation Strategies for beyond-Rule-of-5 Compounds. Adv. Drug Deliv. Rev. 2016, $101,6-21$.

(22) Alhalaweh, A.; Alzghoul, A.; Kaialy, W.; Mahlin, D.; Bergström, C. a S. Computational Predictions of Glass-Forming Ability and Crystallization Tendency of Drug Molecules. Mol. Pharm. 2014, 11, 3123-2132.

(23) Nurzyńska, K.; Booth, J.; Roberts, C. J.; McCabe, J.; Dryden, I.; Fischer, P. M.; Nurzynska, K.; Booth, J.; Roberts, C. J.; McCabe, J.; Dryden, I.; Fischer, P. M. LongTerm Amorphous Drug Stability Predictions Using Easily Calculated, Predicted and Measured Parameters. Mol. Pharm. 2015, 12 (9), 150803143537002.

(24) Mahlin, D.; Bergström, C. A. S. Early Drug Development Predictions of Glass-Forming Ability and Physical Stability of Drugs. Eur. J. Pharm. Sci. 2013, 49 (2), 323-332.

(25) Alzghoul, A.; Alhalaweh, A.; Mahlin, D.; Bergström, C. A. S. Experimental and Computational Prediction of Glass Transition Temperature of Drugs. J. Chem. Inf. Model. 2014, 54 (12), 3396-3403.

(26) Alhalaweh, A.; Alzghoul, A.; Mahlin, D.; Bergström, C. A. S. Physical Stability of Drugs after Storage above and below the Glass Transition Temperature: Relationship to Glass-Forming Ability. Int. J. Pharm. 2015, 495 (1), 312-317.

(27) Gupta, J.; Nunes, C.; Jonnalagadda, S. A Molecular Dynamics Approach for Predicting the Glass Transition Temperature and Plasticization Effect in Amorphous Pharmaceuticals. Mol. Pharm. 2013, 10 (11), 4136-4145.

(28) Christina, B.; Taylor, L. S.; Mauer, L. J. Physical Stability of L-Ascorbic Acid Amorphous Solid Dispersions in Different Polymers: A Study of Polymer Crystallization Inhibitor Properties. Food Res. Int. 2015, 76, 867-877. 
(29) Wegiel, L. A.; Mauer, L. J.; Edgar, K. J.; Taylor, L. S. Crystallization of Amorphous Solid Dispersions of Resveratrol during Preparation and Storage - Impact of Different Polymers. J. Pharm. Sci. 2013, 102 (1), 171-184.

(30) Van den Mooter, G.; Wuyts, M.; Blaton, N.; Busson, R.; Grobet, P.; Augustijns, P.; Kinget, R. Physical Stabilisation of Amorphous Ketoconazole in Solid Dispersions with Polyvinylpyrrolidone K25. Eur. J. Pharm. Sci. 2001, 12, 261-269.

(31) Khougaz, K.; Clas, S.-D. Crystallization Inhibition in Solid Dispersions of MK-0591 and Poly ( Vinylpyrrolidone ) Polymers. J. Pharm. Sci. 2000, 89 (10), 1325-1334.

(32) Edueng, K.; Mahlin, D.; Bergström, C. A. S. The Need for Restructuring the Disordered Science of Amorphous Drug Formulations. Pharm. Res. 2017, 1-19.

(33) ICH Expert Working Group. ICH Guideline Q1A(R2) Stability Testing of New Drug Substances and Products. In International Conference on Harmonization; International conference on harmonisation of techinical requirements for registration of pharmaceuticals for human use, 2003; p 24.

(34) Royal Society of Chemistry. ChemSpider http://www.chemspider.com/ (accessed Jun 11, 2016).

(35) The R foundation. The R Project for Statistical Computing https://www.r-project.org/ (accessed May 1, 2016).

(36) Song, Y.; Yang, X.; Chen, X.; Nie, H.; Byrn, S.; Lubach, J. W. Investigation of DrugExcipient Interactions in Lapatinib Amorphous Solid Dispersions Using Solid-State NMR Spectroscopy. Mol. Pharm. 2015, 12 (3), 857-866.

(37) Thybo, P.; Pedersen, B. L.; Hovgaard, L.; Holm, R.; Mullertz, A. Characterization and Physical Stability of Spray Dried Solid Dispersions of Probucol and PVP-K30. Pharm. Dev. Technol. 2008, 13 (5), 375-386.

(38) Andrews, G. P.; Abudiak, O. A.; Jones, D. S. Physicochemical Characterization of Hot Melt Extruded Bicalutamide-Polyvinylpyrrolidone Solid Dispersion. J. Pharm. Sci. 2010, 99 (3), 1322-1335.

(39) Kalivoda, A.; Fischbach, M.; Kleinebudde, P. Application of Mixtures of Polymeric Carriers for Dissolution Enhancement of Fenofibrate Using Hot-Melt Extrusion. Int. J. Pharm. 2012, 429 (1-2), 58-68.

(40) Ha, E.; Baek, I.; Cho, W.; Hwang, S.; Kim, M. Preparation and Evaluation of Solid Dispersion of Atorvastatin Calcium with Soluplus ${ }^{\circledR}$ by Spray Drying Technique. ... Pharm. Bull. 2014, 62 (June), 545-551.

(41) Taylor, L. S. Physical Stability and Crystallization Inhibition. In Pharmaceutical amorphous solid dispersions; Newman, A., Ed.; John Wiley \& Sons, Inc.: Hoboken, New Jersey, 2015; pp 179-217.

(42) Eerdenbrugh, B. Van; Taylor, L. Small Scale Screening to Determine the Ability of Different Polymers to Inhibit Drug Crystallization upon Rapid Solvent Evaporation. Mol. Pharm. 2010, 7 (4), 1328-1337.

(43) Huang, Y.; Dai, W.-G. Fundamental Aspects of Solid Dispersion Technology for Poorly 
Soluble Drugs. Acta Pharm. Sin. B 2014, 4 (1), 18-25.

(44) Narayan, P.; Porter III, W. W.; Brackhagen, M.; Tucker, C. Polymers and Surfactants. In Pharmaceutical amorphous solid dispersions; Newman, A., Ed.; John Wiley \& Sons, Inc.: Hoboken, New Jersey, 2015; pp 42-84.

(45) BASF. Technical Information Soluplus. BASF Pharma Ingredients \& Services 2010, pp $1-8$.

(46) Ashland. AquaSolve and AquaSolve AS Hydroxypropylmethylcellulose Acetate Succinate Physical and Chemical Properties Handbook. Ashland 2013.

(47) Bühler, V. Kollidon VA64. In Kollidon - Polyvinylpryrrolidone excipients for the Pharmaceutical Industry; BASF, 2008; pp 207-254.

(48) Li, Y.; Pang, H.; Guo, Z.; Lin, L.; Dong, Y.; Li, G.; Lu, M.; Wu, C. Interactions between Drugs and Polymers Influencing Hot Melt Extrusion. J. Pharm. Pharmacol. 2014, 66 (2), 148-166.

(49) Rumondor, A. C. F.; Taylor, L. S. Effect of Polymer Hygroscopicity on the Phase Behavior of Amorphous Solid Dispersions in the Presence of Moisture. Mol. Pharm. 2009, 7 (2), 477-490.

(50) Vasanthavada, M.; Tong, W. Q.; Joshi, Y.; Kislalioglu, M. S. Phase Behavior of Amorphous Molecular Dispersions I: Determination of the Degree and Mechanism of Solid Solubility. Pharm. Res. 2004, 21 (9), 1598-1606.

(51) Pharmaceutical Amorphous Solid Dispersions; Newman, A., Ed.; John Wiley \& Sons, Inc.: Hoboken, New Jersey, 2015.

(52) Janssens, S.; Zeure, A. De; Paudel, A.; Van Humbeeck, J.; Rombaut, P.; Van Den Mooter, G. Influence of Preparation Methods on Solid State Supersaturation of Amorphous Solid Dispersions: A Case Study with Itraconazole and Eudragit E100. Pharm. Res. 2010, 27 (5), 775-785.

(53) Tian, Y.; Caron, V.; Jones, D. S.; Healy, A.-M.; Andrews, G. P. Using Flory-Huggins Phase Diagrams as a Pre-Formulation Tool for the Production of Amorphous Solid Dispersions: A Comparison between Hot-Melt Extrusion and Spray Drying. J. Pharm. Pharmacol. 2014, 66 (2), 256-274.

(54) Singh, A.; De Bisschop, C.; Schut, H.; Van Humbeeck, J.; Van Den Mooter, G. Compression Effects on the Phase Behaviour of Miconazole-Poly (1-VinylpyrrolidoneCo-Vinyl Acetate) Solid Dispersions - Role of Pressure, Dwell Time, and Preparation Method. J. Pharm. Sci. 2015, 104 (10), 3366-3376.

(55) Baird, J. a.; Taylor, L. S. Evaluation of Amorphous Solid Dispersion Properties Using Thermal Analysis Techniques. Adv. Drug Deliv. Rev. 2012, 64 (5), 396-421.

(56) Yoshioka, M.; Hancock, B. C.; Zografi, G. Crystallization of Lndomethacin from the Amorphous State below and above Its Glass Transition Temperature. J. Pharm. Sci. 1994, 83 (12), 1700-1705.

(57) Mahlin, D.; Ponnambalam, S.; Heidarian Höckerfelt, M.; Bergström, C. A. S. Toward In Silico Prediction of Glass-Forming Ability from Molecular Structure Alone: A 
Screening Tool in Early Drug Development. Mol. Pharm. 2011, 8 (2), 498-506.

(58) Baird, J. A.; Eerdenbrugh, B. Van; Taylor, L. S. A Classification System to Assess the Crystallization Tendency of Organic Molecules from Undercooled Melts. J. Pharm. Sci. 2010, 99 (9), 3787-3806.

(59) Wang, R.; Pellerin, C.; Lebel, O. Role of Hydrogen Bonding in the Formation of Glasses by Small Molecules: A Triazine Case Study. J. Mater. Chem. 2009, 19, 2747.

(60) Moore, M. D.; Wildfong, P. L. D. Informatics Calibration of a Molecular Descriptors Database to Predict Solid Dispersion Potential of Small Molecule Organic Solids. Int. J. Pharm. 2011, 418 (2), 217-226. 